\title{
Mathematical Modelling of Plankton-Oxygen Dynamics under the Climate Change
}

\author{
Yadigar Sekerci \& Sergei Petrovskii ${ }^{1}$ \\ Department of Mathematics, University of Leicester, \\ University Road, Leicester LE1 7RH, U.K.
}

\begin{abstract}
Ocean dynamics is known to have a strong effect the global climate change and on the composition of the atmosphere. In particular, it is estimated that about $70 \%$ of the atmospheric oxygen is produced in the oceans due to the photosynthetic activity of phytoplankton. However, the rate of oxygen production depends on water temperature and hence can be affected by the global warming. In this paper, we address this issue theoretically by considering a model of a coupled plankton-oxygen dynamics where the rate of oxygen production slowly changes with time to account for the ocean warming. We show that a sustainable oxygen production is only possible in an intermediate range of the production rate. If, in the course of time, the oxygen production rate becomes too low or too high, the system's dynamics changes abruptly resulting in the oxygen depletion and plankton extinction. Our results indicate that the depletion of atmospheric oxygen on global scale (which, if happens, obviously can kill most of life on Earth) is another possible catastrophic consequence of the global warming, a global ecological disaster that has been overlooked.
\end{abstract}

Keywords: phytoplankton, global warming, oxygen depletion, extinction, pattern formation

\footnotetext{
${ }^{1}$ Corresponding author. Phone/fax: +44 116252 3916/3915, email: sp237@le.ac.uk
} 


\section{Introduction}

Global warming has been an issue of huge debate and controversy over the last decade because of its potential numerous adverse effects on ecology and society [44]. Ocean makes about two-thirds of the Earth surface and also works as a huge heat capacitor, and hence is expected to be heavily affected by the climate change. Discussion of the effect of warming on the ocean dynamics usually focuses on possible changes in the global circulation [74] and on the expected melting of polar ice resulting in the consequential flooding on the global scale [61, 62]. Meanwhile, apart from the apparent hydrophysical aspects of the problem, ocean is also a huge ecosystem and the effect of global warming on its functioning may have disastrous consequences comparable or even worse than the global flooding.

Functioning of marine ecosystems, with a particular interest in the plankton dynamics, has long been a focus in ecological and environmental research [33, 46, 76]. One reason for this is that plankton is the basis of the marine food chain; hence, a good understanding of its dynamics is required for more efficient fishery [16, 22, 57]. In a more general context, understanding of plankton dynamics is important for a reliable estimation of marine ecosystems productivity [5, 38]. We mention here that mathematical modelling has proved to be an efficient research tool to reveal the properties of the plankton dynamics using both simple, conceptual models and more complicated 'realistic' ones [3, 5, 26, 57, 64, 66, 77].

Plankton is not only a key element of the marine food web but also have a significant effect on the climate [11, 78] and the composition of the atmosphere, in particular, on the amount of oxygen [33, 60]. Plankton consists of two different taxa such as phytoplankton and zooplankton. Zooplankton are animals (e.g. krill) and phytoplankton are plants. As most plants do, phytoplankton can produce oxygen in photosynthesis when sufficient light is available, e.g. in the photic layer of the ocean during the daytime. The oxygen first come to the water and eventually into the air through the sea surface thus contributing to the total oxygen budget in the atmosphere. This contribution appears to be massive: it is estimated that about $70 \%$ of the Earth atmospheric oxygen is produced by the ocean phytoplankton [33, 60]. Correspondingly, one can expect that a decrease in the rate of the oxygen production by phytoplankton may have catastrophic consequences for life on Earth possibly resulting in mass extinction of animal species, including the mankind. Therefore, identification of potential threats to the oxygen production is literally an issue of vital importance.

It is well known that the water temperature has a notable effect on the phytoplankton growth [4, 22, 72]. Because of its control on phytoplankton metabolic processes, the temperature can be expected to affect the oxygen production and this has indeed been observed in several studies [13, 46, 50]. Phytoplankton produces oxygen in photosynthesis during the day but consumes it through respiration during the night. The surplus - i.e. the difference (per phytoplankton cell) between the amount of oxygen produced during the 
day and its amount consumed during the night - is called the net oxygen production. It is the net production that eventually contributes to the atmospheric oxygen. The matter is that the photosynthesis rate and the respiration rate depend on the water temperature differently [32, 73]. As a result, the net production is a function of temperature. The temperature dependence of the net production observed in the sea was attributed to the effect of local geophysical anomalies [73]. In this paper, we consider an increase in the water temperature resulting form the global warming. Our goal is to understand what can be the effect of the water warming on the coupled plankton-oxygen dynamics and how it may affect the oxygen budget.

We address the above problem by means of mathematical modelling. Firstly, we develop a conceptual plankton-oxygen model that takes into account basic processes related to oxygen production in photosynthesis and its consumption, because of respiration, by phyto- and zooplankton in a well-mixed (nonspatial) system. Secondly, we consider the properties of the model (both analytically and numerically) and show that the system's dynamics is only sustainable in a certain parameter range, namely, for an intermediate value of the oxygen production rate. The corresponding long-term system behavior is determined either by a positive "coexistence" stable steady state or by a stable limit cycle. Outside that range, the system does not possess positive attractors and the dynamics is not sustainable resulting in plankton extinction and oxygen depletion. Thirdly, we consider the system's response to a gradual change in the oxygen production rate (the latter being assumed to be a result of the global warming) and show that, once the production rate surpasses a certain critical value, the system goes to extinction.

We then consider a spatially explicit extension of our model where plankton and oxygen are carried around by turbulent water flows. In the parameter range where the nonspatial system possesses a stable limit cycle, the spatial system exhibits the formation of irregular spatiotemporal patterns. The patterns are self-organized in the sense that they are not caused by any pre-defined spatial structure. We show that the response of the spatial system to the gradual change in the oxygen production rate is similar to that of the nonspatial one, i.e. a sufficiently large change in the production rate results in the plankton extinction and oxygen depletion. Interestingly, the spatial system appears to be sustainable in a broader parameter range so that plankton and oxygen persist for values of the production rate where the nonspatial system goes extinct. We also show that the spatial system may provide some early-warning signals for the approaching ecological disaster (i.e. plankton extinction and oxygen depletion); in particular, we observe that, prior to the disaster, the spatial distributions of plankton and oxygen become almost periodical. 


\section{Main Equations}

Marine ecosystem is a complex system consisting of many nonlinearly interacting species, organic substances and inorganic chemical components. Correspondingly, a 'realistic' ecosystem model can consist of many equations [24, 48]. However, since in this paper we are mostly interested in the dynamics of the dissolved oxygen (for the sake of brevity, the word 'dissolved' will be omitted below), in particular in the conditions of sustainable oxygen production due to the phytoplankton photosynthetic activity, such a detailed description seems to be excessive. Instead, we consider a simple, conceptual model that includes oxygen itself and the phytoplankton as its producer. The model also include zooplankton as the effect of zooplankton on the oxygen-phytoplankton dynamics is expected to be important for two reasons: (i) because it is known to be a factor controlling the phytoplankton density [33, 77] and (ii) the zooplankton obviously consumes oxygen through breathing. The structure of the model is shown schematically in Fig. 1.

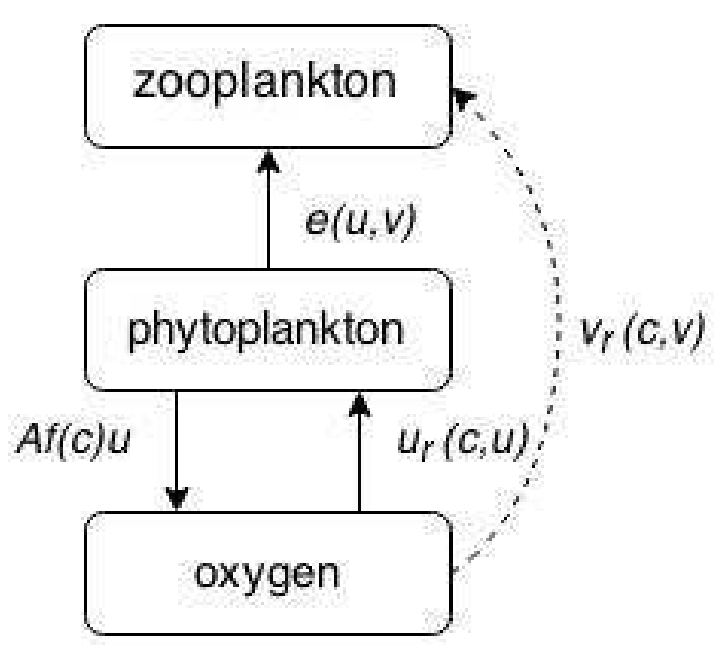

Figure 1: The structure of our conceptual model describing the interactions between oxygen, phytoplankton and zooplankton. Arrows show flows of matter through the system. Phytoplankton produces oxygen through photosynthesis during the day-time and consumes it during the night. Zooplankton feeds on phytoplankton and consumed oxygen through breathing; more details are given in the text.

Differential equations are known to be an efficient mathematical framework to describe the plankton dynamics [24, 39, 40, 41]. We begin with the non-spatial case which is relevant in the case of a well-mixed ecosystem. The corresponding model is given by the 
following equations:

$$
\begin{aligned}
& \frac{d c}{d t}=A f(c) u-u_{r}(c, u)-v_{r}(c, v)-m c \\
& \frac{d u}{d t}=g(c, u)-e(u, v)-\sigma u \\
& \frac{d v}{d t}=\kappa(c) e(u, v)-\mu v
\end{aligned}
$$

where $c$ is the concentration of oxygen at time $t, u$ and $v$ are the densities of phytoplankton and zooplankton, respectively. The term $A f(c)$ describes the rate of oxygen production per unit phytoplankton mass. Whilst function $f(c)$ describes the rate of increase in the concentration of the dissolved oxygen because of its transport from phytoplankton cells to the surrounding water (see the details below), factor $A$ takes into account the effect of the environmental factors (such as the water temperature) on the rate of oxygen production inside the cells. Function $g(c, u)$ describes the phytoplankton growth rate which is known to be correlated to the rate of photosynthesis [26]; we therefore assume it to depend on the amount of available oxygen. Terms $u_{r}$ and $v_{r}$ in Eq. (1) are the rates of oxygen consumption by phytoplankton and zooplankton, respectively, because of their respiration. The coefficient $m$ is the rate of oxygen loss due to the natural depletion (e.g. due to biochemical reactions in the water). The term $e$ in Eq. (2) describes feeding of zooplankton on phytoplankton. The consumed phytoplankton biomass is transformed into the zooplankton biomass with efficiency $\kappa$, see the first term in the right-hand side of Eq. (3). Since the well-being of zooplankton obviously depend on the oxygen concentration (so that, ultimately, it dies if there is not enough oxygen to breathe), we assume that $\kappa=$ $\kappa(c)$. Coefficients $\sigma$ and $\mu$ are the natural mortality rates of the phyto- and zooplankton, respectively.

In order to understand what can be the properties of function $f(c)$, we have to look more closely at the oxygen production. Oxygen is produced inside phytoplankton cells in photosynthesis and then diffuses through the cell membrane to the water. Diffusion flux always directed from areas with higher concentration of the diffusing substance to the the areas with lower ones and it is the larger the larger is the difference between the concentrations (cf. the Fick law). Therefore, for the same rate of photosynthesis, the amount of oxygen that gets through the cell membrane will be the larger the lower is the oxygen concentration in the surrounding water. Therefore, $f$ should be a monotonously decreasing function of $c$ that tends to zero when the oxygen concentration in the water is becoming very large, i.e. for $c \rightarrow \infty$. The above features are qualitatively taken into account by the following parametrization:

$$
f(c)=1-\frac{c}{c+c_{0}}=\frac{c_{0}}{c+c_{0}},
$$

(known as the Monod kinetics) where $c_{0}$ is the half-saturation constant.

Considering phytoplankton multiplication, we assume that $g(c, u)=\alpha(c) u-\gamma u^{2}$ where the first term describes the phytoplankton linear growth and the second term account for 
intraspecific competition. Here $\alpha(c)$ is the per capita growth rate and coefficient $\gamma$ describes the intensity of the intraspecific competition. Phytoplankton produce oxygen in photosynthesis (during the day) but it also needs it for breathing (mainly during the night), therefore we assume that low oxygen concentration is unfavorable for phytoplankton and is likely to depress its reproduction. On the other hand, a phytoplankton cell cannot take more oxygen than it needs. Hence $\alpha$ should be a monotonously increasing function of $c$ tending to a constant value for $c \rightarrow \infty$. The simplest parametrization for $\alpha$ is then given by the Monod function:

$$
\alpha(c)=\frac{B c}{c+c_{1}},
$$

where $B$ is the maximum phytoplankton per capita growth rate in the large oxygen limit and $c_{1}$ is the half-saturation constant. Thus, for $g(c, u)$ we obtain:

$$
g(c, u)=\frac{B c u}{c+c_{1}}-\gamma u^{2}
$$

Note that, in the special case $c=$ const, Eq. (1) can me omitted and the system (1-3) reduces to a prey-predator system with the logistic growth for prey (phytoplankton):

$$
\begin{aligned}
\frac{d u}{d t} & =(K(c)-u) \gamma u-e(u, v)-\sigma u \\
\frac{d v}{d t} & =\kappa(c) e(u, v)-\mu v,
\end{aligned}
$$

where the carrying capacity $K(c)=(B / \gamma) c /\left(c+c_{1}\right)$ appears to be a function of oxygen concentration. We mention here that the prey-predator system has often been used in plankton studies either to study some fundamental properties of the plankton dynamics [57] or as an elementary 'building block' in more complicated models [48]. We consider Holling type II response for the predator (zooplankton) and use the following standard parametrization for predation:

$$
e(u, v)=\frac{\beta u v}{u+h}
$$

where $\beta$ is the maximum predation rate and $h$ is the half-saturation prey density.

The next step is to decide about the parametrization of plankton respiration, see the second and third terms in the right-hand side of Eq. (1). For the phytoplankton respiration, we apply the argument similar to the one used above for the phytoplankton growth rate $\alpha(c)$ and describe it as

$$
u_{r}(c, u)=\frac{\delta u c}{c+c_{2}},
$$

where $\delta$ is the maximum per capita phytoplankton respiration rate and $c_{2}$ is the halfsaturation constant. 
Regarding the zooplankton respiration, for many zooplankton species their oxygen consumption is known to depend on the oxygen concentration [12, 13, 17]. The consumption rate usually shows a linear increase at small oxygen concentration but tends to a constant, saturating value at large oxygen concentration (cf. Fig. 1 in [12]). The simplest parametrization of this kinetics is the Monod function:

$$
v_{r}(c, u)=\frac{\nu c v}{c+c_{3}}
$$

where $\nu$ is the maximum per capita zooplankton respiration rate and $c_{3}$ is the halfsaturation constant.

Finally, with regard to the zooplankton feeding efficiency as a function of the oxygen concentration, $\kappa(c)$, there are generic biological arguments suggesting that it should be of sigmoidal shape, i.e. being approximately constant for the oxygen concentrations above a certain threshold but promptly decaying to zero for the concentration below the threshold [42, 71]. Correspondingly, we parameterize it as follows:

$$
\kappa(c)=\frac{\eta c^{2}}{c^{2}+c_{4}^{2}}
$$

where $0<\eta<1$ is the maximum feeding efficiency and $c_{4}$ is the half-saturation constant.

Considering Eqs. (1-3) together with (4), (6) and (9-12), our model takes the following more specific form:

$$
\begin{aligned}
\frac{d c}{d t} & =\frac{A c_{0} u}{c+c_{0}}-\frac{\delta u c}{c+c_{2}}-\frac{\nu c v}{c+c_{3}}-m c \\
\frac{d u}{d t} & =\left(\frac{B c}{c+c_{1}}-\gamma u\right) u-\frac{\beta u v}{u+h}-\sigma u \\
\frac{d v}{d t} & =\frac{\eta c^{2}}{c^{2}+c_{4}^{2}} \frac{\beta u v}{u+h}-\mu v .
\end{aligned}
$$

Due to their biological meaning, all parameters are nonnegative.

Since Eqs. (13-15) will mostly be studied by simulations, it is convenient to introduce dimensionless variables. Let us consider

$$
t^{\prime}=t m, \quad c^{\prime}=\frac{c}{c_{0}}, \quad u^{\prime}=\frac{\gamma u}{m}, \quad v^{\prime}=\frac{\beta v}{m}
$$

and the new (dimensionless) parameters as

$$
\begin{aligned}
& \hat{B}=\frac{B}{m}, \quad \hat{A}=\frac{A}{c_{0} \gamma}, \quad \hat{\delta}=\frac{\delta m}{c_{0} \gamma}, \quad \hat{\nu}=\frac{\nu m}{\beta c_{0}}, \quad \hat{\sigma}=\frac{\sigma}{m}, \quad \hat{\mu}=\frac{\mu}{m}, \\
& \hat{h}=\frac{\gamma h}{m}, \quad \hat{\eta}=\frac{\eta \beta}{m}, \quad \text { and } \quad \hat{c_{i}}=\frac{c_{i}}{c_{0}} \quad \text { where } \quad i=1,2,3,4 .
\end{aligned}
$$


Equations (13-15) then turn into the following:

$$
\begin{aligned}
\frac{d c}{d t} & =\frac{A u}{c+1}-\frac{\delta u c}{c+c_{2}}-\frac{\nu c v}{c+c_{3}}-c \\
\frac{d u}{d t} & =\left(\frac{B c}{c+c_{1}}-u\right) u-\frac{u v}{u+h}-\sigma u \\
\frac{d v}{d t} & =\frac{\eta c^{2}}{c^{2}+c_{4}^{2}} \frac{u v}{u+h}-\mu v
\end{aligned}
$$

where primes and hats are omitted for the notations simplicity. Note that meaningful range of dimensionless parameters is, generally speaking, different from that of the original (dimensional) parameters; in particular, the dimensionless feeding efficiency $\eta$ is not necessarily less than one any more.

In the next section, we consider the steady states of the system (19-21), and its transient dynamics will be considered in Section 3.3. A spatially-explicit extension of the system (19 21) will be considered in Section 5 .

\section{Analysis and Preliminary Results}

System (19-21) is rather complicated. In order to better understand it properties, it is instructive to start the analysis with a simpler model that does not contain zooplankton, which is obtained from $(19-21)$ by setting $v(t) \equiv 0$.

\subsection{Oxygen-phytoplankton system}

The zooplankton-free model is given by the following equations:

$$
\begin{aligned}
\frac{d c}{d t} & =\frac{A u}{c+1}-\frac{u c}{c+c_{2}}-c \\
\frac{d u}{d t} & =\left(\frac{B c}{c+c_{1}}-u\right) u-\sigma u .
\end{aligned}
$$

Correspondingly, its steady states are the solutions of the following system:

$$
\begin{aligned}
\frac{A u}{c+1}-\frac{u c}{c+c_{2}}-c & =0 \\
\left(\frac{B c}{c+c_{1}}-u\right) u-\sigma u & =0 .
\end{aligned}
$$

Equations (24-25) define the two (null)isoclines of the system, which we will call the oxygen isocline and the phytoplankton isocline, respectively. The oxygen isocline is therefore given by

$$
u=\frac{c(c+1)\left(c+c_{2}\right)}{A c_{2}+(A-1) c-c^{2}}
$$




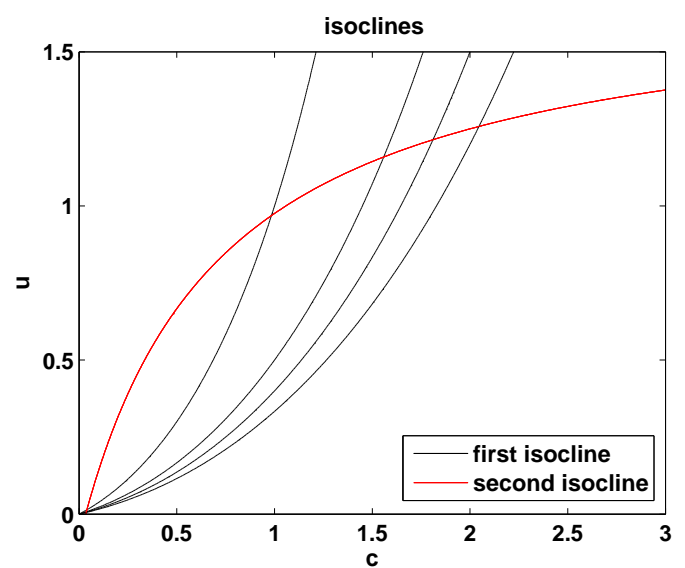

(a)

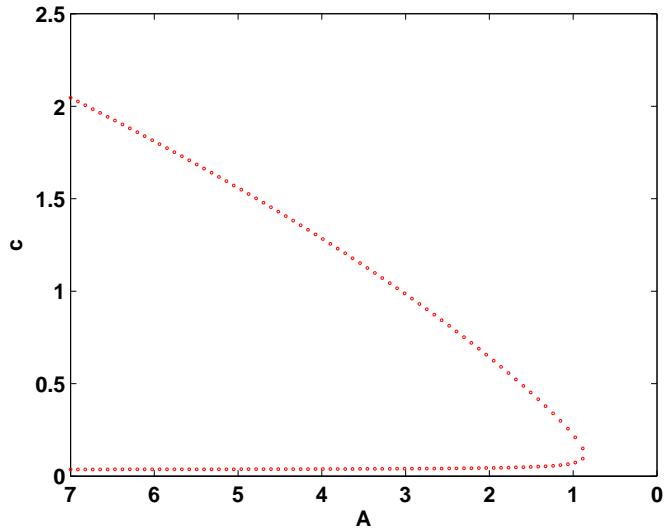

(b)

Figure 2: (a) The (null)isoclines of the oxygen-phytoplankton system (24-25). Black curves show the first (oxygen) isocline (26) obtained for $c_{2}=1$ and $A=3,5,6$ and 7 (from left to right, respectively); red curve shows the second (phytoplankton) isocline (27) obtained for $B=1.8, \sigma=0.1$ and $c_{1}=0.7$. (b) The steady state values of $c$ (obtained as the intersection points of the two isoclines) as a function of the controlling parameter $A$.

The phytoplankton isocline consists of two parts, i.e. of the following curve

$$
u=\frac{B c}{c+c_{1}}-\sigma \quad(u>0)
$$

and the straight line $u=0$.

We readily observe that $(0,0)$ is a steady state of the system. As for the positive equilibria (if any), it does not seem possible to solve Eqs. (26-27) explicitly. Instead, since system's equilibria are the intersection points of the two isoclines, important conclusions can be made by analyzing the mutual position of the corresponding curves. Figure $2 \mathrm{a}$ shows the isoclines (26) and (27) obtained for some hypothetical parameter values. Therefore, system (24-25) can have two positive equilibria $\left(\tilde{c}_{1}, \tilde{u}_{1}\right)$ and $\left(\tilde{c}_{2}, \tilde{u}_{2}\right)$.

It is readily seen that the extinction state $(0,0)$ exists for all parameter vales. The two positive equilibria only exist in a certain parameter range. Considering $A$ as the controlling parameter (for the reason that will be explained in Section 4) and taking into account its effect on the shape of the oxygen isocline (cf. Fig. 2a), we conclude that the positive equilibria are only feasible if $A$ is not too small, i.e. above a certain critical value. When the value of $A$ decreases, the two positive steady states move towards each other, so that they eventually merge and disappear; see Fig. $2 \mathrm{~b}$.

The stability of the steady states can be revealed by considering the direction of the flow $\left(\frac{d c}{d t}, \frac{d u}{d t}\right)$ in the $(c, u)$ phase plane of the system $(22-23)$; see Fig. 3 . It is straightforward to see that the extinction state and the upper positive state are stable nodes whilst 


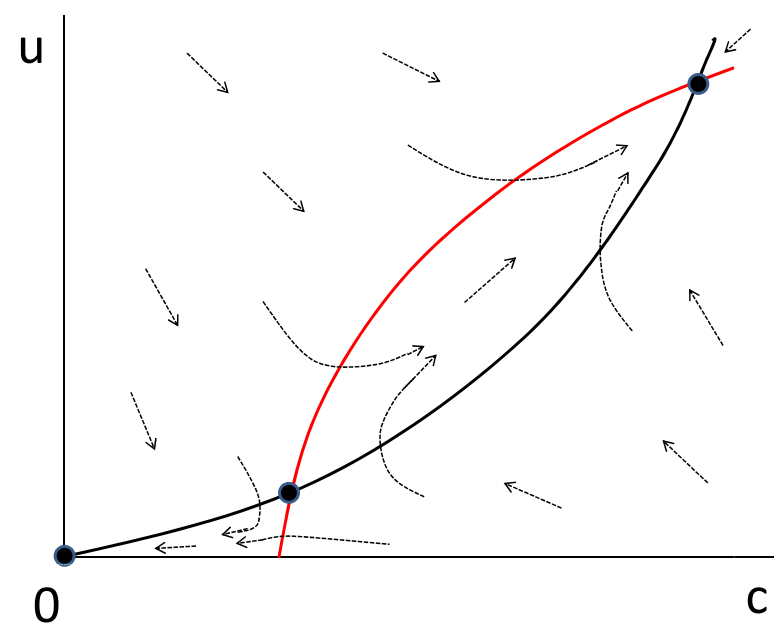

Figure 3: A sketch of the phase plane of the oxygen-phytoplankton system. Black and red curves show the oxygen isocline and phytoplankton isocline, respectively, large black dots show the steady states, black dashed curves show sample trajectories of the system, arrows show the direction of the phase flow as given by vector $\left(\frac{d c}{d t}, \frac{d u}{d t}\right)$.

the lower positive state is a saddle (hence unstable). Thus, when $A$ falls below the critical value, the disappearance of the positive steady states happens in the saddle-node bifurcation, so that only trivial the extinction state remains feasible. A similar change in the system properties (i.e. the disappearance of the positive equilibria in a saddlenode bifurcation) can also occur as a result of an increase in $c_{1}$ or decrease in $B$. From the ecological point of view, this bifurcation corresponds to an ecological disaster with a complete depletion of oxygen and the extinction of phytoplankton.

\subsection{Oxygen-phyto-zooplankton system: steady states analysis}

We now proceed to the analysis of the equilibria of the full system (19-21). Obviously, the steady state values are the solutions of the following equations:

$$
\begin{aligned}
\frac{d c}{d t}=0 & =\frac{A u}{c+1}-\frac{u c}{c+c_{2}}-\frac{\nu c v}{c+c_{3}}-c \\
\frac{d u}{d t}=0 & =\left(\frac{B c}{c+c_{1}}-u\right) u-\frac{u v}{u+h}-\sigma u \\
\frac{d v}{d t}=0 & =\frac{\eta c^{2}}{c^{2}+c_{4}^{2}} \frac{u v}{u+h}-\mu v .
\end{aligned}
$$

Correspondingly, the system possesses the following steady states:

- The trivial equilibrium $E_{1}=(0,0,0)$ corresponding to the depletion of oxygen and the extinction of plankton. It is readily seen that this equilibrium exists always, regardless the choice of parameter values. 
- The two semi-trivial, boundary equilibria $E_{2}^{(1)}=\left(\tilde{c}_{1}, \tilde{u}_{1}, 0\right)$ and $E_{2}^{(2)}=\left(\tilde{c}_{2}, \tilde{u}_{2}, 0\right)$ where the steady state values $\tilde{c}_{i}$ and $\tilde{u}_{i}(\mathrm{i}=1,2)$ are given by Eqs. $(26-27)$. Correspondingly, as was discussed in Section 3.1, these states exist only if $A$ is above the critical value.

- The positive (coexistence) equilibrium $E_{3}=(\bar{c}, \bar{u}, \bar{v})$.

Note that, whilst the existence of steady states $E_{1}, E_{2}^{(1)}$ and $E_{2}^{(2)}$ can be readily seen, the existence and uniqueness of $E_{3}$ is not at all obvious. System (28-30) cannot be solved analytically. Instead, Eqs. (28-30) were solved numerically with parameter values chosen from a broad range, and it was established that a positive steady state exists unless $A$ is too large or too small.

Now we discuss the stability of steady states $E_{1}, E_{2}^{(1)}, E_{2}^{(2)}$ and $E_{3}$. A general method to reveal the stability of a steady state is to calculate the eigenvalues of the Jacobian matrix (see Appendix). For the extinction state $\left(E_{1}\right)$, calculations are simple resulting in $\lambda_{1}=-1, \lambda_{2}=-\sigma$ and $\lambda_{3}=-\mu$. Thus, the extinction state is always stable.

For the boundary state $E_{2}^{(1)}$, we recall that the corresponding steady state $\left(\tilde{c}_{1}, \tilde{u}_{1}\right)$ of the oxygen-phytoplankton subsystem is a saddle; therefore, $E_{2}^{(1)}$ is unstable.

In order to reveal the stability of $E_{2}^{(2)}$, we consider the characteristic equation of the corresponding Jacobian matrix:

$$
\begin{aligned}
& {\left[\left(-\frac{A u}{(1+c)^{2}}-1-\frac{u c_{2}}{\left(c+c_{2}\right)^{2}}-\lambda\right)\left(\frac{B c}{c+c_{1}}-2 u-\sigma-\lambda\right)\right.} \\
& \left.\quad-\left(\frac{A}{1+c}-\frac{c}{c+c_{2}}\right)\left(\frac{B c_{1} u}{\left(c+c_{1}\right)^{2}}\right)\right] \cdot\left(\frac{u}{u+h}\left(\frac{c^{2}}{c^{2}+c_{4}^{2}}\right)-\mu-\lambda\right)=0,
\end{aligned}
$$

where $c$ and $u$ are the steady state values given by Eqs. (26-27). Due to the structure of the Jacobian matrix (see Appendix), Eq. (31) factorizes so that the part in the square brackets corresponds to the oxygen-phytoplankton subsystem where we already know the result: $\left(\tilde{c}_{2}, \tilde{u}_{2}\right)$ is always stable, therefore both eigenvalues are negative. The stability of $E_{2}^{(2)}$ is then determined by the third eigenvalue:

$$
\lambda_{3}=\frac{u}{u+h}\left(\frac{c^{2}}{c^{2}+c_{4}^{2}}\right)-\mu .
$$

Unfortunately, Eq. (32) is not instructive because $c$ and $u$ are not known explicitly. In order to reveal the stability of $E_{2}^{(2)}$, we have to analyze it numerically; the results are shown in Fig. 4 below.

Regarding the stability of the coexistence steady state $E_{3}$, the corresponding characteristic equation is very complicated and hence can only be solved numerically. We obtain that, for this steady state, one eigenvalue is real and always negative whilst the other two eigenvalues are complex conjugate where their real part can be positive or negative depending on parameter values. 
Figure 4 summarizes the results of the steady states analysis as a map in parameter plane $\left(A, c_{1}\right)$. Here $c_{1}$ is chosen as the second controlling parameter because it describes the effect of oxygen on phytoplankton growth. In Domain 3, i.e. for some intermediate values of $A, E_{3}$ is stable and $E_{2}^{(2)}$ is unstable. The system therefore exhibits bistability (recall that $E_{1}$ is always stable). With a decrease in $A, E_{2}^{(2)}$ becomes stable, so that for the parameters in Domain 2 the system exhibits tristability. With a further decrease in $A$, i.e. in Domain $1, E_{2}^{(2)}$ disappears in a saddle-node bifurcation (see Section 3.1). For approximately the same value of $A$, the coexistence state disappears as well so that, for sufficiently small values of $A$, the only attractor of the system is the extinction state.

With an increase in $A$, i.e. in Domain $4, E_{2}^{(2)}$ remains unstable (a saddle point) and $E_{3}$ looses its stability to become an unstable focus. For these parameters, the unstable state $E_{3}$ is surrounded by a stable limit cycle that appears through the Hopf bifurcation when crossing from Domain 3 to Domain 4. With a further increase in $A$, the limit cycle disappears through a nonlocal bifurcation when crossing from Domain 4 to Domain 5 . For parameter values from Domain 5, the only attractor of the system is the extinction state $E_{1}$.

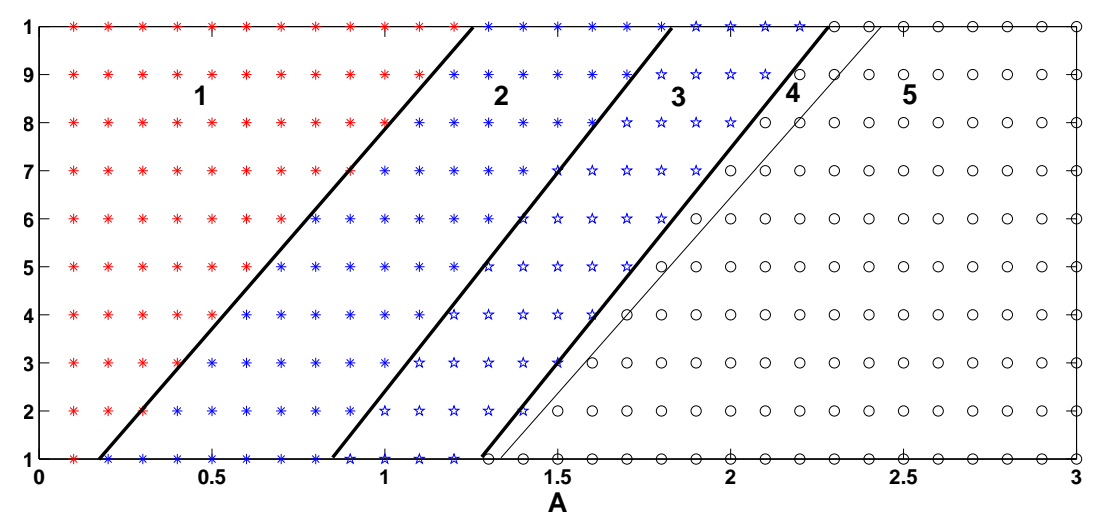

Figure 4: $\mathrm{A}$ map in the parameter plane $\left(A, c_{1}\right)$ where different domains correspond to different stability of equilibria $E_{2}^{(2)}$ and $E_{3}$; see details in the text. Since the map is obtained in numerical simulations, the position of the domains boundaries is approximate.

From the ecological point of view, the structure of $\left(A, c_{1}\right)$ parameter plane means that the system is viable (in particular, ensuring sustainable production of oxygen) only in the intermediate range of $A$. As we will show below, this observation have important implications in the context of the climate change.

\subsection{Numerical simulations}

In the nonspatial system (19-21), the information about the steady states existence and stability provides an exhausting overview of the large-time dynamics of the system. However, it does not always give enough details about the transient stage of the dynamics 
given by the evolution of specific initial conditions. Meanwhile, there is a growing understanding that the transient dynamics may be more relevant to the dynamics of real ecosystems than the large-time asymptotics, cf. [34, 35]. In order to make an insight into this issue, in this section we present the results of numerical simulations of the system (19.21) choosing parameter values representative of all interesting dynamical regimes. We fix most of the parameters at some hypothetical values as $B=1.8, \sigma=0.1, c_{1}=0.7$, $c_{2}=1, c_{3}=1, c_{4}=1, \eta=0.7, \delta=1, \nu=0.01, \mu=0.1$ and $h=0.1$, but vary $A$ in a broad range.

Figure 5 shows the oxygen and plankton densities versus time obtained for parameter $A$ chosen in Domain 4 or Domain 5 (cf. Fig. 4). For parameters of Fig. 5 a, the system possesses a stable limit cycle so that the initial conditions promptly converge to oscillatory dynamics. Parameters of Fig. $5 \mathrm{~b}$ are further away from the Hopf bifurcation curve (i.e. the boundary between Domains 3 and 4) so that the limit cycle is of a bigger size, which

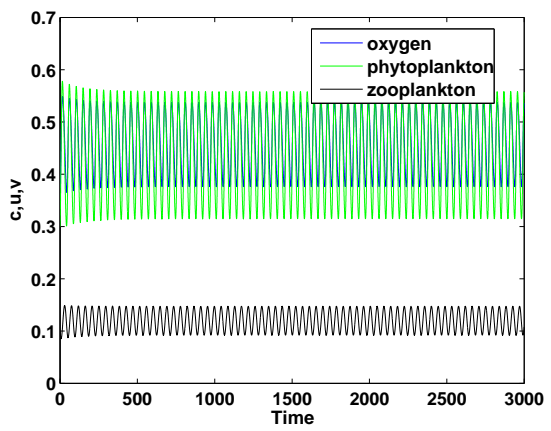

(a)

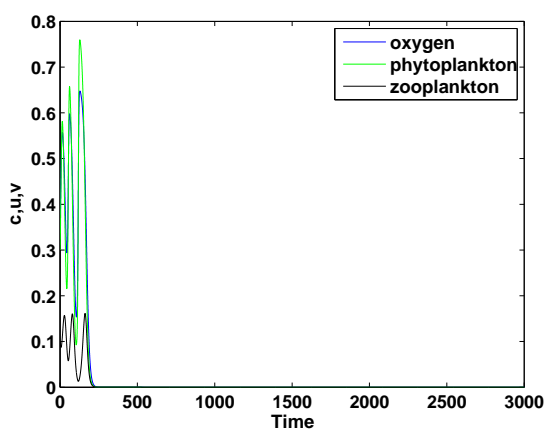

(c)

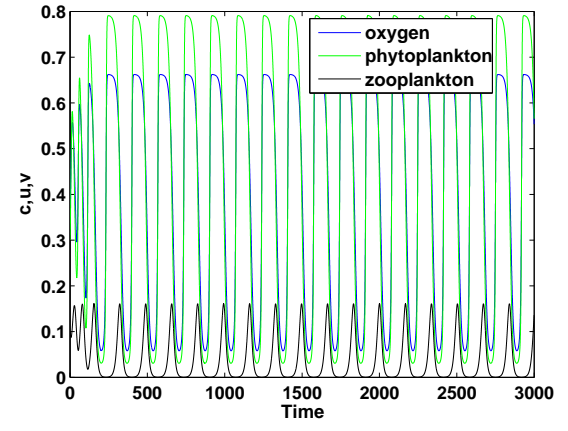

(b)

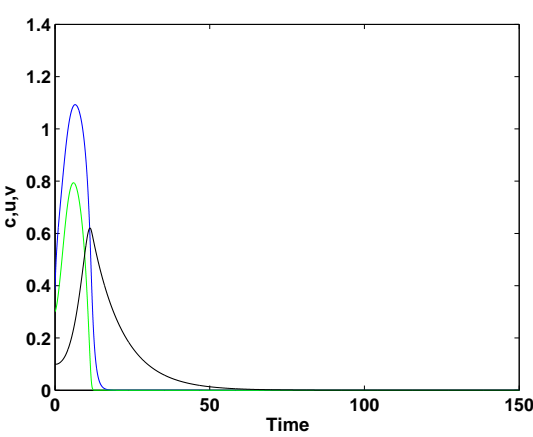

(d)

Figure 5: Oxygen concentration (blue) and phyto- and zooplankton densities (green and black, respectively) vs time obtained for the initial conditions $c_{0}=0.385, u_{0}=0.3$, $v_{0}=0.1$ and parameters (a) $A=2.02$, (b) $A=2.0534$, (c) $A=2.054$, and (d) $A=4$. Other parameters are given in the text. 


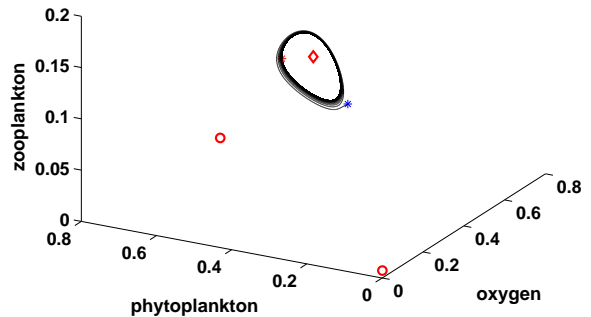

(a)

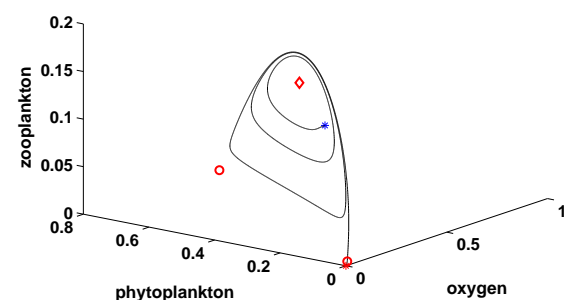

(c)

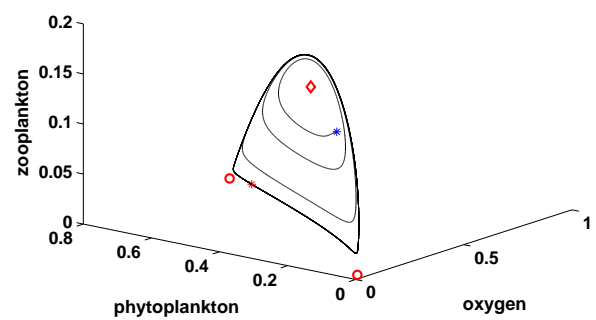

(b)

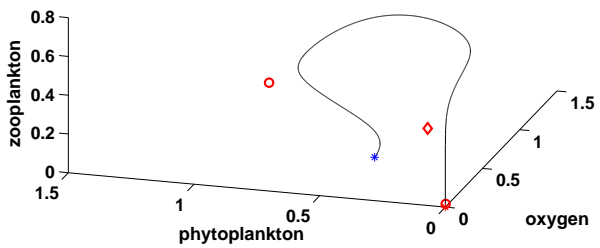

(d)

Figure 6: Trajectories of the oxygen-phyto-zooplankton dynamical system shown in the corresponding 3D phase space. Blue star shows the starting point (i.e. the initial values), red star shows the end point reached over the given time. Red diamond shows the coexistence steady state $E_{3}$, red circles show the zooplankton-free states $E_{2}^{(1)}$ and $E_{2}^{(2)}$. Parameters are the same as in Fig. $\underline{5}$.

results in sustainable oscillations of a much larger amplitude. The limit cycle disappears for parameters of Fig. $5 \mathrm{c}$, so that the species densities go to extinction after just a few oscillations. Parameters of Fig. 5d are further into Domain 5, there is no limit cycle and it is not surprising that the initial conditions promptly converge to zero. Interestingly, the extinction is preceded by an outbreak, so that the initial values first show a significant increase before starting decaying to extinction.

In order to make it easier to understand the system dynamics, as well as to trace the changes in the system properties, we also use another way to present the simulation results. Figure 6 shows the results of Fig. $[5$ as the trajectories in the corresponding threedimensional phase space. Here the red circles show the boundary steady states $E_{2}^{(1)}$ and $E_{2}^{(2)}$, the red diamond shows the positive coexistence steady state $E_{3}$, the blue star shows the initial conditions, i.e. the starting point of the trajectory, and the red star shows the end point of the trajectory reached over the given simulation time. It readily seen that 
the peak in the species densities observed in Fig. $5 \mathrm{~d}$ is the effect of the saddle point $E_{2}^{(1)}$.

We mention here that, in the parameter range where the stable limit cycle exists, the system has two attractors: the limit cycle itself and the extinction state $E_{1}$. Hence the dynamics depends on the initial conditions, i.e. which basin of attraction they belong to. For the results shown in Figs. $5 a-b$ and $\underline{6} a-b$, the initial conditions were chosen from the attraction basin of the limit cycle. In case the initial condition are chosen in the attraction basin of $E_{1}$, the dynamics is less interesting as the trajectories approach the origin without any oscillations (not shown here).

\section{Effect of the global warming: results and preliminary discussion}

As was discussed in the introduction, variations in the local water temperature can have an effect on the photosynthesis rate and hence on the net amount of oxygen produced over the daily cycle [32, 73]. In our model (19-21) (or (13-15) in the original dimensional variables), the rate of oxygen production is quantified by parameter $A$. In order to reflect the effect of temperature $T$ on photosynthesis, $A$ becomes a function of $T$. In its turn, the temperature is a function of time, so that $A$ becomes a function of time too. Therefore, we consider $A=A(t)$ but keep other parameters fixed for the sake of simplicity.

Water temperature is known to fluctuate significantly on all temporal scales, e.g. hourly, daily, monthly and annually [43]. A "realistic" function $A(t)$ taking into account those fluctuations is likely to be very complicated. However, since the purpose of this study is to consider the effect of global warming conceptually rather than predictively, this level of details seems to be excessive. Instead, in order to account for the general trend (and not for details), we consider the simplest possible choice of $A(t)$, i.e. the linear function:

$$
A=A_{0} \quad \text { for } \quad t<t_{1}, \quad A=A_{0}+\omega\left(t-t_{1}\right) \quad \text { for } \quad t \geq t_{1} .
$$

Here $t_{1}$ is the moment when the global warming started, $A_{0}$ is the rate of net oxygen production 'before changes', and parameter $\omega$ quantifies the rate of global warming.

Available data are meagre and it remains unclear what is the typical phytoplankton response to an increase in the water temperature, i.e. whether the rate of oxygen production by phytoplankton actually decreases or increases. Therefore, we consider two possible scenarios: (i) where a higher water temperature facilitates oxygen production $(\omega>0)$ and (ii) where a higher temperature hampers oxygen production $(\omega<0)$. Since global warming is a slow process, we consider $\omega$ to be very small, i.e. $|\omega| \ll \min \{A, B, \delta, \sigma, \mu, \nu \eta\}$. For the initial value of $A$, we assume that prior to the climate change the ecosystem was in a 'safe state', i.e. with the coexistence steady state $E_{3}$ either being stable (Domain 3 in Fig. 4) or unstable but surrounded by a stable limit cycle (Domain 4), so that $A_{0}$ is chosen accordingly. 
Note that, with $A$ now being a function of $t$, the system (19 21) becomes nonautonomous and strictly speaking the results of the previous section do not immediately apply. However, having assumed that $A(t)$ is a slow changing function, we expect that the properties of the corresponding autonomous system (i.e. with $A=$ const) in different parameter ranges (see Fig. 4) can provide a convenient skeleton for understanding the effect of changes.

We begin with case (i), i.e. $\omega>0$. Based on the heuristic arguments above, the system is expected to develop, in the course of time, oscillations of increasing amplitude as an increase in $A$ eventually moves the system further into Domain 4 , hence further away from the Hopf bifurcation curve. If the warming continues for a sufficiently long time, which results in $A$ becoming sufficiently large, in one can expect that all species should go extinct (in particular, the oxygen concentration drops to zero) once the system moves to Domain 5 where the only steady state of the autonomous system is extinction.

This appears to be in full agreement with simulations. Figure 7 shows the oxygen concentration and plankton densities versus time obtained for the rate of warming $\omega=$ $10^{-5}$ and two different initial values of the oxygen production rate, Figs. 7 a,c for $A_{0}=1.97$ and Figs. $7 \mathrm{~b}, \mathrm{~d}$ for $A_{0}=2$. In both cases, $A_{0}$ is in the parameter range where $E_{3}$ is a stable focus (Domain 3). Correspondingly, in both cases, at early times the system exhibits oscillations of decreasing amplitude as the system is converging to the stable steady state. In the case shown in Figs. $7 \mathrm{a}$,c, the final value of the oxygen production rate is $A=2$ which is still below the Hopf bifurcation value (which is $A_{H} \approx 2.01$ ) so that the oscillations eventually die out. However, in the case shown in Fig. 7b,d, the final value of the oxygen production rate is $A=2.03>A_{H}$. Correspondingly, although the amplitude of the oscillations decreases at the beginning, it starts increasing when the system passes the Hopf bifurcation point.

For parameter values that result in a larger final value of $A$, i.e. either a larger $A_{0}$ or a larger $\omega$, the changes in the system dynamics can be more significant. Figure 8 shows the oxygen concentration and the plankton densities vs time obtained for the same rate of warming $\omega=10^{-5}$ but larger initial values of the oxygen production rate, $A_{0}=2.024$ and $A_{0}=2.048$. In both cases, $A_{0}$ lies in the parameter range where $E_{3}$ is a unstable focus surrounded by a stable limit cycle (Domain 4). For $A_{0}=2.024$ (see Fig. 8 8 a,c), the warming leads to oscillations of steadily growing amplitude but without any qualitative changes in the dynamics. However, for $A_{0}=2.048$ (Fig. 8b,d), the increase in $A$ results in an ecological disaster where, after a number of oscillations of increasing amplitude, plankton suddenly goes extinct and the oxygen concentration falls to zero. This sudden and dramatic change in the dynamics obviously happens when $A$ moves, in the course of time, to the parameter range where there is no limit cycle and the only attractor of the corresponding autonomous system is extinction (cf. Domain 5 in Fig. 4).

A similar dramatic change in the system dynamics can occur for a smaller, 'safe' value of $A_{0}$ but for a higher rate of global warming. Figure 9 shows the oxygen concentration 


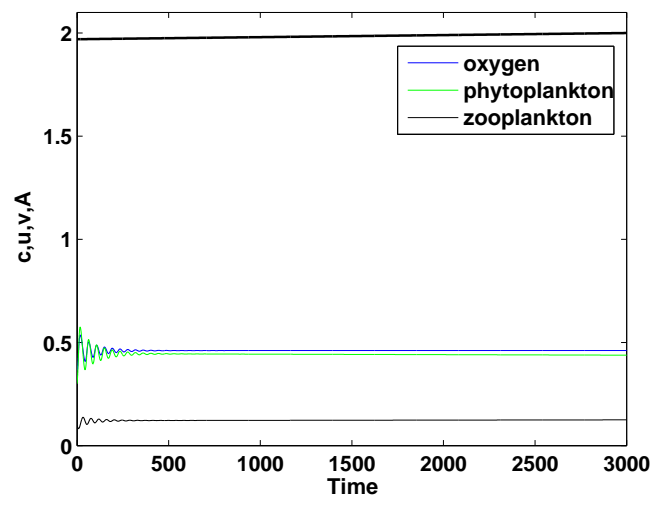

(a)

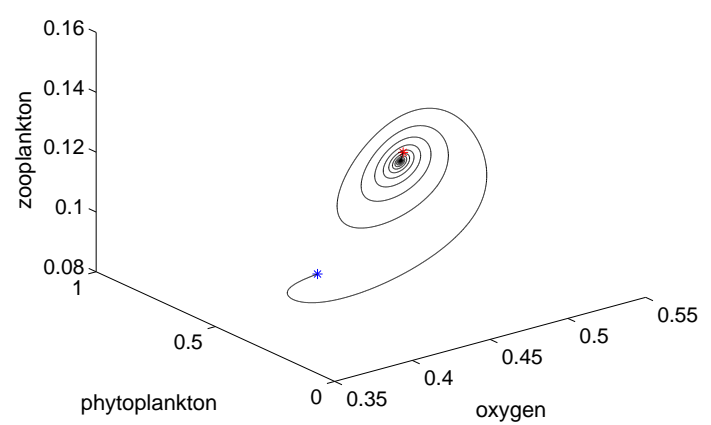

(c)

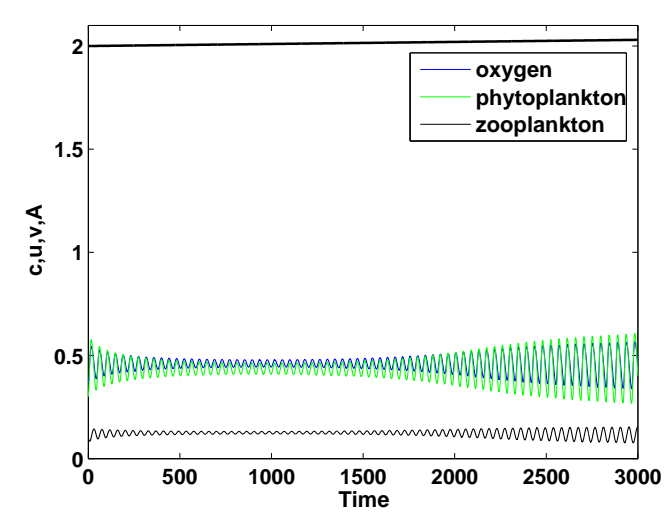

(b)

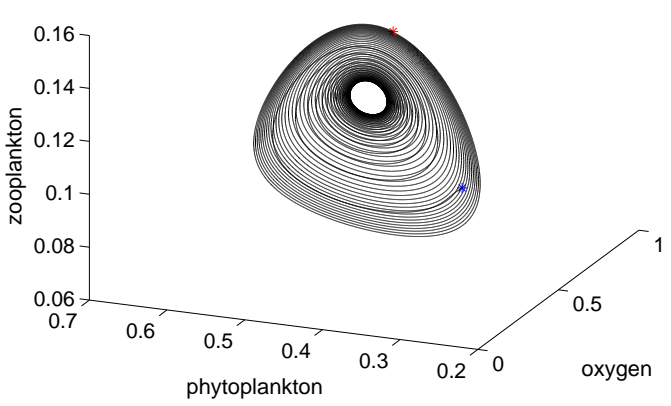

(d)

Figure 7: (a,b) Oxygen concentration (blue) and the density of phyto- and zooplankton (green and black, respectively) vs time obtained for $\omega=10^{-5}$ and the initial conditions $c_{0}=0.385, u_{0}=0.3$ and $v_{0}=0.1$, the thick black line at the top shows $A(t) ;(\mathrm{c}, \mathrm{d})$ the corresponding system trajectories shown in the 3D phase space of the system. (a,c) for $A_{0}=1.97,(\mathrm{~b}, \mathrm{~d})$ for $A_{0}=2$, other parameters are given in the text (see the beginning of Section 3.3 .

and plankton densities obtained for $A_{0}=2$ (as in Figs. 7b,d) and $\omega=10^{-4}$. It is readily seen that the effect of climate changes on the dynamics of the system follows the same scenario where the system exhibits oscillations of increasing amplitude up to a certain time but then suddenly go to extinction (oxygen depletion). A close look at $A(t)$ (shown by the thick black line) reveals that the disaster occurs for approximately the same value of $A$ as in Fig. $8 \mathrm{~b}$, i.e. for $A \approx 2.055$.

Note that, since in our conceptual model (33) the global warming is assumed to continue indefinitely, the change in the system dynamics resulting in the catastrophic plank- 


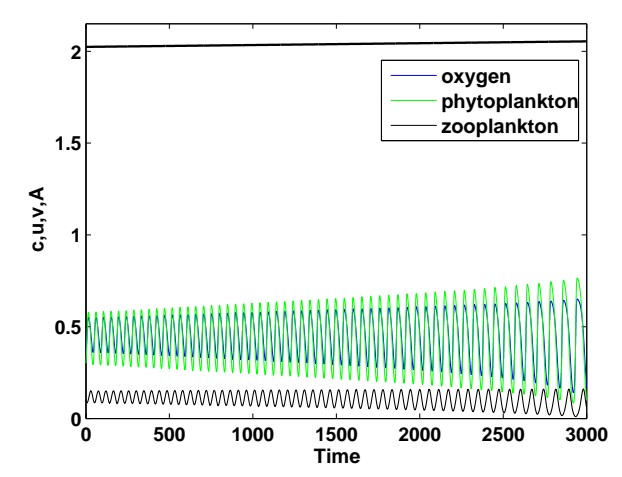

(a)

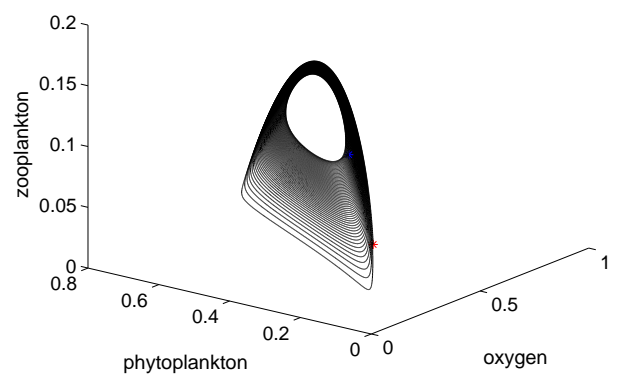

(c)

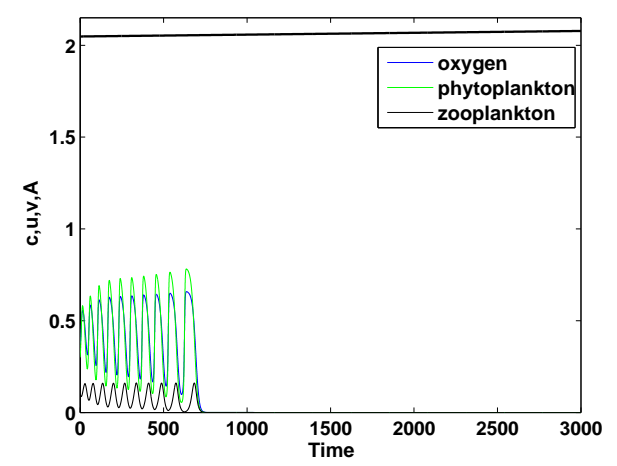

(b)

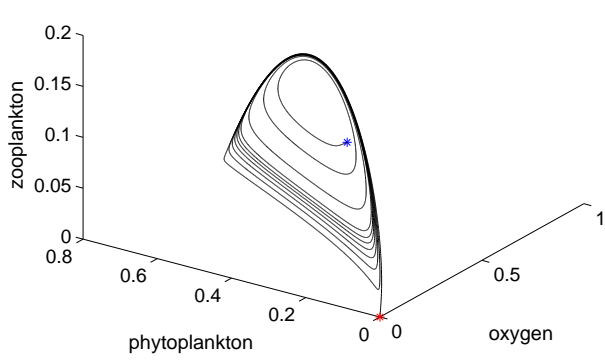

(d)

Figure 8: (a,b) Oxygen concentration (blue) and the density of phyto- and zooplankton (green and black, respectively) vs time; (c,d) the corresponding system trajectories in the 3D phase space of the system. (a,c) for $A_{0}=2.024$, (b,d) for $A_{0}=2.048$, other parameters are the same as in Fig. 7 .

ton extinction and oxygen depletion is bound to happen regardless the initial conditions and the rate of change $\omega$. A choice of smaller $A_{0}$ or $\omega$ would only require a longer time for $A(t)$ to reach the dangerous range but could not avert the disaster. In particular, none of the cases shown in Figs. 7 and 8 a,c is sustainable; in each of these cases, if the system is let to run for longer, it will inevitably end up in extinction.

We now consider case (ii) where an increase in water temperature hampers the oxygen production, i.e. where $\omega<0$. As we have seen above, the effect of changes in the oxygen production rate $A$ on the system dynamics can be predicted and understood by establishing the correspondence between the value of $A(t)$ at different time and the properties of the system with $A=$ const at different ranges of parameter $A$ (see Fig. 4). Therefore, one can expect that the plankton should go extinct and the oxygen concentration to drop to zero once $A$ becomes sufficiently small, i.e. when moving to Domain 1 where the only 


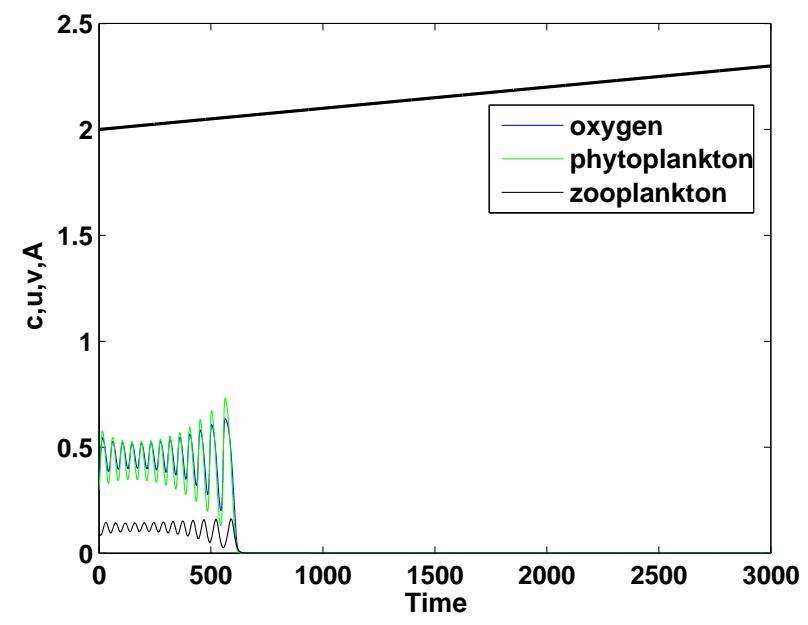

Figure 9: Oxygen concentration (blue) and the density of phyto- and zooplankton (green and black, respectively) vs time obtained for $A_{0}=2$ and $\omega=10^{-4}$, other parameters are the same as above. Thick black line shows $A(t)$.

steady state of the autonomous system is extinction. This appears to be in full agreement with simulations (that we do not show here for the sake of brevity). The dynamics is somewhat trivial in this case. Concentration $c$ and densities $u$ and $v$ remain close to their steady state values (as given by $E_{3}$ ); no oscillations were observed. At the moment when $A(t)$ reaches the critical value where $E_{3}$ disappears, the densities start decreasing and promptly approach the extinction state $E_{1}$. As well as in case (i), the extinction occurs regardless the initial conditions and the rate of warming $\omega$; a choice of large $A_{0}$ and/or smaller $\omega$ only postpone the extinction but cannot avert it.

Therefore, we arrive at the conclusion that sustainable functioning of the planktonoxygen system is only possible in a certain intermediate range of the oxygen production rate $A$. Since $A$ is known to be a function of temperature, it suggests that sustainable functioning is only possible in an intermediate range of temperatures. Even if the current state of the system is safe, a sufficiently large warming (roughly estimated as $5-6^{\circ} \mathrm{C}[73]$ ) would inevitably lead to an ecological disaster resulting in a complete depletion of oxygen.

The above conclusion is made based on the properties of our model (19-21). The question arises here as to how general and realistic this conclusion is as the model apparently leaves many features of real marine ecosystems out of the scope. In particular, the model (19 21) does not contain space, hence assuming that both oxygen and plankton are distributed uniformly. Although this assumption is known to work well in some cases (in the so-called "well-mixed" systems), it becomes irrelevant in a situation where the non-uniformity of the plankton distribution becomes prominent and hence cannot be neglected. Meanwhile, strongly heterogeneous spatial distribution of plankton is rather common in marine ecosystems [23, 56, 76]. An observation that makes the spatial aspect of the plankton dynamics especially relevant to this study is that conditions of species 
extinction can be significantly different in spatially-explicit models compared to their nonspatial counterparts; see [67, 68, 69]. Because of the self-organized pattern formation, the species may be able to persist in a parameter range where they would go extinct in the nonspatial system [54]. In the next section, we consider a spatially-explicit version of the model (19-21) and investigate how space may alter the effect of global warming on the plankton dynamics and the oxygen production.

\section{Spatial Dynamics}

One factor that makes the ecosystem dynamics spatial is the movement of organisms. In the marine environment, movement occurs because organisms either are entrained with the turbulent water flows or possess the ability to self-motion, or because of a combined action of the two mechanism. Phytoplankton is mostly passive but zooplankton can move by itself. The relative importance of turbulent flows and self-motion depends on the spatial scale and the spatial structure of the aquatic environment. For instance, because of ocean stratification and the relatively small thickness of the upper (productive) ocean layer, vertical migration of zooplankton is known to be very important and strongly affects its vertical distribution and feeding rates [29]. On the contrary, horizontal self-motion is thought to play a minor role compared to the effect of lateral turbulent mixing [70].

Correspondingly, for the purposes of this study we consider the following idealized system. We assume that the vertical distribution of both phyto- and zooplankton is uniform through the photic layer where most of the photosynthetic activity takes place. For the horizontal distribution, we consider a one-dimensional (1D) space, which can be interpreted as a transect along the 2D ocean surface. Oxygen concentration and plankton densities are functions of space $x$ and time $t$, i.e. $c(x, t), u(x, t)$ and $v(x, t)$. We neglect the effect of zooplankton self-movement and consider the horizontal turbulence as the main mechanisms of spatial transport. The turbulent mixing is known to be approximated well by the turbulent diffusion [59, 64]; thus, from (19-21) we arrive at the following equations:

$$
\begin{aligned}
\frac{\partial c}{\partial t} & =D_{T} \frac{\partial^{2} c}{\partial x^{2}}+\frac{A u}{c+1}-\frac{\delta u c}{c+c_{2}}-\frac{\nu c v}{c+c_{3}}-c \\
\frac{\partial u}{\partial t} & =D_{T} \frac{\partial^{2} u}{\partial x^{2}}+\left(\frac{B c}{c+c_{1}}-u\right) u-\frac{u v}{u+h}-\sigma u \\
\frac{\partial v}{\partial t} & =D_{T} \frac{\partial^{2} v}{\partial x^{2}}+\frac{\eta c^{2}}{c^{2}+c_{4}^{2}} \frac{u v}{u+h}-\mu v .
\end{aligned}
$$

Here $D_{T}$ is the turbulent diffusion coefficient and $0<x<L$ where $L$ is the size of the domain.

For numerical simulations, it is convenient to introduce the dimensionless coordinate as $x^{\prime}=x \sqrt{m / D_{T}}$ in line with our choice of other dimensionless variables; see the end of Section 2. In terms of the system $(34-36)$, it means that $D_{T}=1$. We emphasize 
that this is not a special choice of the turbulent diffusion coefficient but just a technical consequence of our choice of dimensionless variables.

Equations (34-36) are solved by the finite differences using the zero-flux boundary conditions. The results below are obtained for the mesh steps $\Delta x=0.5$ and $\Delta t=0.01$; we checked that these values are small enough not to bring numerical artifacts.

Since we are mostly interested in conditions of sustainable oxygen production and this, in our model, is quantified by parameter $A$, and also because the system properties were shown to depend on $A$ significantly, we consider the dynamics of the the system (34-36) for different values of $A$ but having other parameters fixed at some hypothetical values as $B=1.8, \sigma=0.1, c_{1}=0.7, c_{2}=1, c_{3}=1, c_{4}=1, \eta=0.7, \delta=1, \nu=0.01, \mu=0.1$, $h=0.1$ and $L=1000$.

The choice of the initial conditions is a subtle issue. An arbitrary choice that is not consistent with the system inherent properties may result in a very long transient dynamics [65]. Combined with the effect of the global warming, cf. Eq. (33), the long-living transients may result in a biased prediction about the system properties. Therefore, in order to generate appropriate initial conditions, we first consider the following "tentative" nearly uniform species distribution:

$$
c(x, 0)=\bar{c}, \quad u(x, 0)=\bar{u}, \quad v(x, 0)=\left(x-\frac{L}{2}\right) \frac{\epsilon}{L}+\bar{v},
$$

were $\bar{c}, \bar{u}$ and $\bar{v}$ are the steady state values (as given by the positive equilibrium $E_{3}$, see Section 3.2 ) and $\epsilon$ is an auxiliary parameter.

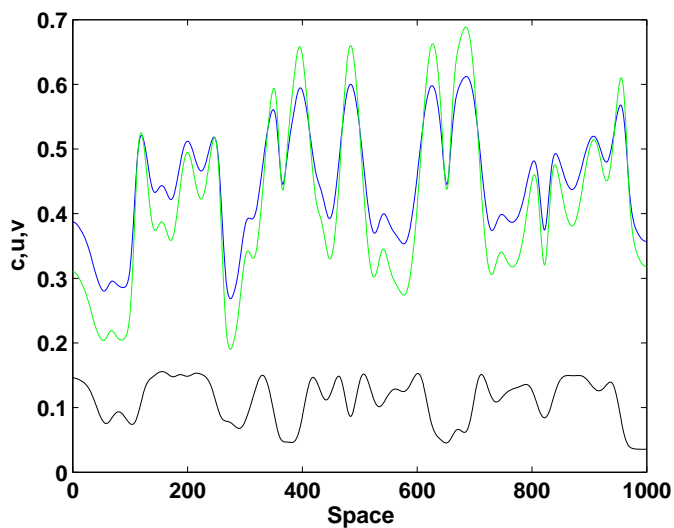

(a)

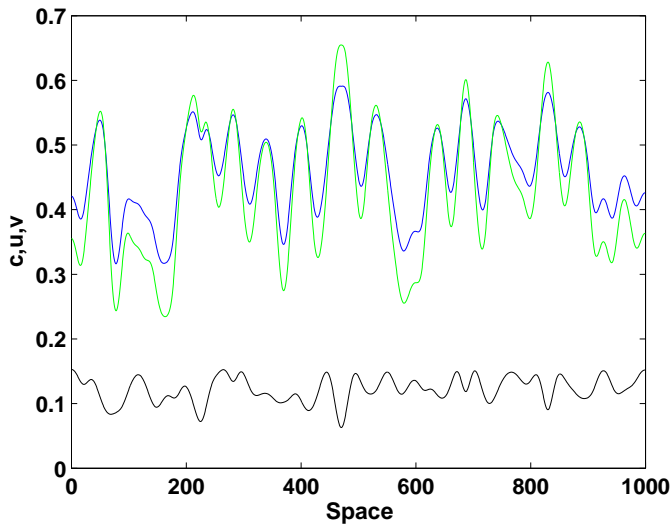

(b)

Figure 10: Snapshots of the oxygen and plankton distribution over space at (a) $t=10000$ and (b) $t=12000$ obtained for the "tentative" initial conditions (37) and parameters $A=2.05$ and $\epsilon=0.02$. For this value of $A$, the local dynamics is oscillatory due to a stable limit cycle. Other parameters are given in the text. 
It is known for other relevant diffusion-reaction systems that initial conditions (37) result in pattern formation which is reflective of system's complex dynamics [54, 57, 65]. This appears to be true for the system $(34-36)$ as well. Figure 10 shows the spatial distributions of oxygen and plankton obtained from (37) after the system (34-36) is run for sufficiently long time to let the transient die out. Note that there is no any notable qualitative difference between the distributions shown in Fig. 10a (obtained for $t=10000$ ) and Fig. 10b (obtained for $t=12000$ ), which indicates that the system has reached its dynamical equilibrium. Correspondingly, we consider distribution shown in Fig. 10a as "inherent" and use it as the initial condition for other simulations shown below.

We now proceed to simulations for different values of $A$. Figure 11a shows the snapshot of oxygen and plankton spatial distributions obtained at $t=10000$ for $A=2.02$. For this value of $A$, the local dynamics is oscillatory; see Fig. $5 \mathrm{a}$. The system dynamics is sustainable and the irregular spatiotemporal pattern persists; no extinction occurs. The amplitude of the spatial variation becomes somewhat smaller compared to the initial condition shown in Fig. 10a, because the size of the limit cycle is smaller for this value of A.

Figure 11b shows the oxygen and plankton distributions obtained at $t=10000$ for $A=2.054$. Again we observe that, in a manner apparently similar to the above, the system persists through formation of spatiotemporal patterns. However, there is one important difference: we recall that, for this value of $A$, the nonspatial system goes to extinction; see Fig. 5c. We therefore conclude that the conditions of sustainable system's functioning are less restrictive in the spatially explicit case.

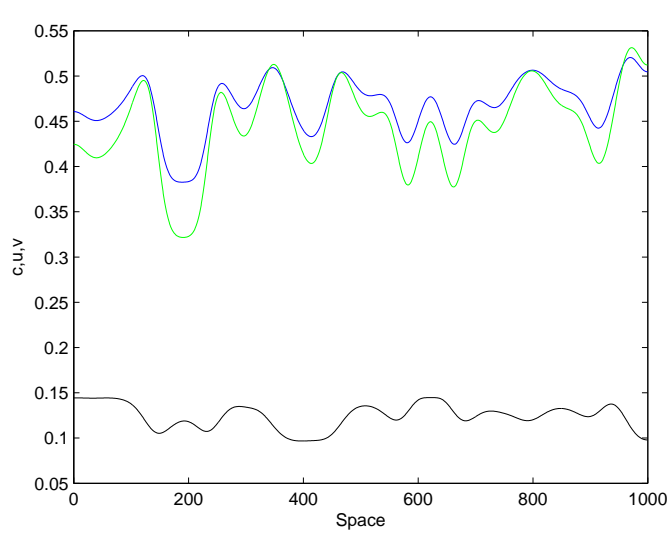

(a)

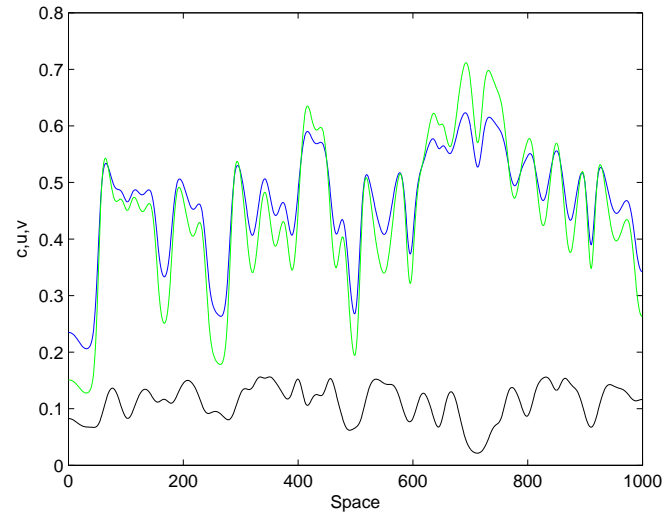

(b)

Figure 11: Snapshots of the oxygen and plankton spatial distribution obtained at $t=$ 10000 for (a) $A=2.02$ and (b) $A=2.054$. Other parameters are given in the text. The initial conditions are shown in Fig. 10a.

The above results were obtained for a fixed value of $A$. Now we are going to consider 
the effect of the global warming as is modeled by Eq. (33), i.e. where $A$ changes with time. Figure 12 shows snapshots of the oxygen and plankton distributions at $t=10000$ obtained for $\omega=10^{-5}$ and different values of the initial oxygen production rate $A_{0}$, Fig. 12a for $A_{0}=2$ and Fig. 12b for $A_{0}=2.048$. The system remains sustainable and no extinction occur. Interestingly, the corresponding 'final' values of $A(t)$ are, respectively, $A=2.1$ and $A=2.148$, which in both cases is larger than the maximum possible $A(t)$ in the nonspatial system: recall that, for the parameters of Fig. $12 \mathrm{~b}$, the nonspatial system goes extinct already at $t \approx 750$, cf. Fig. $8 \mathrm{~b}$. However, a further increase in $A$, i.e. if the system is let to run for a longer time, results in the system crash (i.e. extinction of plankton and complete depletion of oxygen) also in the spatial system. Figure 13 shows the plankton densities and oxygen concentration vs time obtained for parameters of Fig. 8b, Fig. 13a shows the local densities (concentration) obtained at a fixed point in the middle of the domain, i.e. $c\left(x_{0}, t\right), u\left(x_{0}, t\right)$ and $v c\left(x_{0}, t\right)$ for $x_{0}=500$, and Fig. $13 \mathrm{~b}$ shows the spatially averaged densities $\langle c\rangle(t),\langle u\rangle(t)$ and $\langle v\rangle(t)$ where

$$
<p>=\frac{1}{L} \int_{0}^{L} p(x, t) d x
$$

where $p=c, u, v$. It is readily seen that gradual decrease of the average densities is followed by a sudden catastrophe when the oxygen production rate becomes too high.

Interestingly, as a response to an increase in $A$, the properties of the species spatial distribution can change significantly in the course of time. Figure 14 shows the snapshots of oxygen and plankton distribution at two different moments obtained for $A_{0}=2.05$ and for $\omega=10^{-4}$. Whilst at the beginning of the warming (see Fig. 14a obtained for

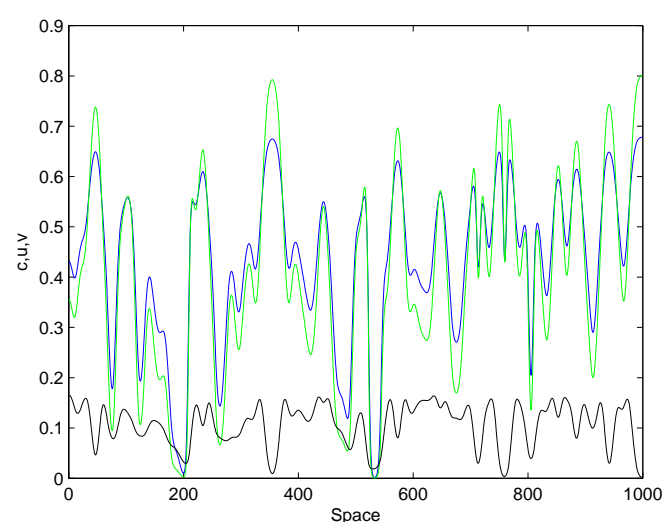

(a)

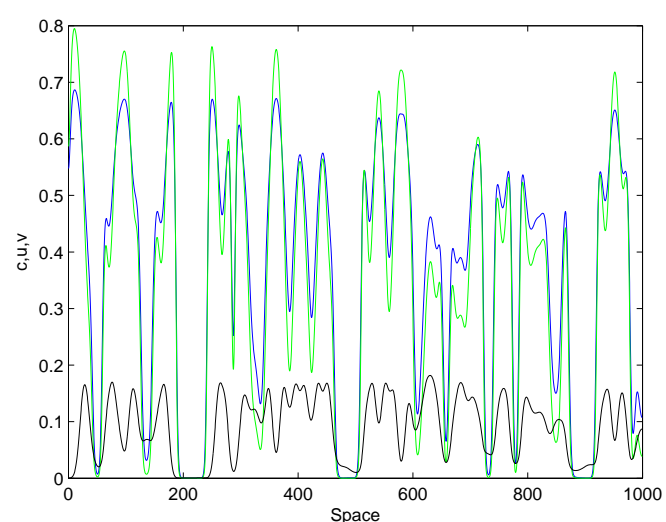

(b)

Figure 12: Snapshots of the oxygen and plankton spatial distribution obtained at $t=$ 10000 for $A(t)$ given by Eq. (33) with $\omega=10^{-5}$ and (a) $A_{0}=2$, (b) $A_{0}=2.048$. Other parameters are given in the text. 


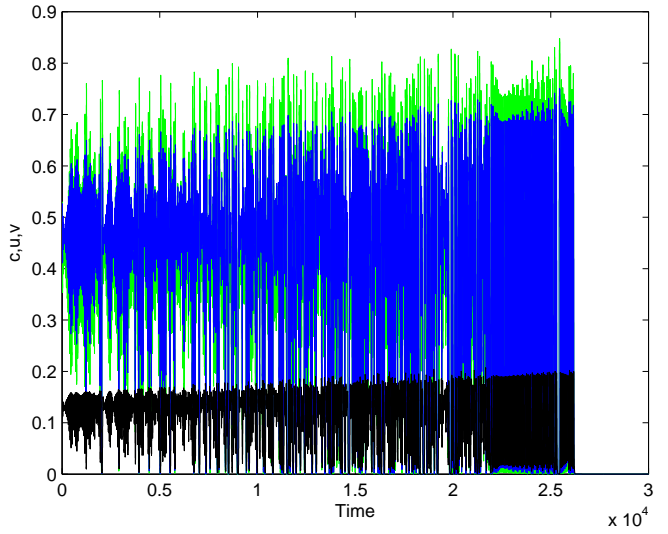

(a)

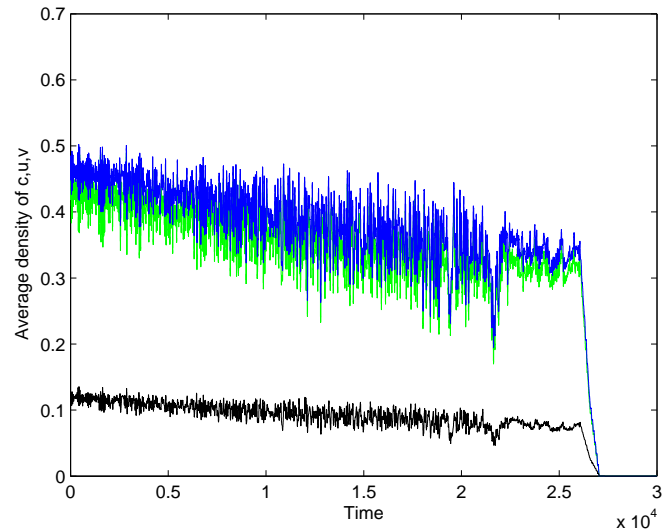

(b)

Figure 13: (a) System components distributions for fixed space $x_{0}=500$ for $t=30000$. (b) System components integration vs. time. The system parameters are taken from Figure $12 b$.

$t=200)$ the spatial distribution remains qualitatively similar to the initial conditions (cf. Fig. 10a), in particular being prominently irregular, at a later time the distribution becomes almost spatially periodical; see Fig. 14b obtained for $t=2950$. Remarkably, for this time $A(t) \approx 2.35$, which is far beyond the safe parameter range of the corresponding nonspatial system (cf. Fig. 5). We therefore observe that the spatial system is more

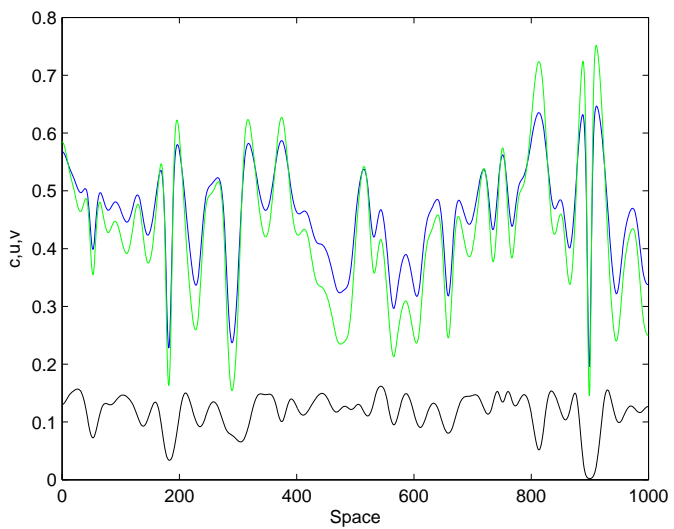

(a)

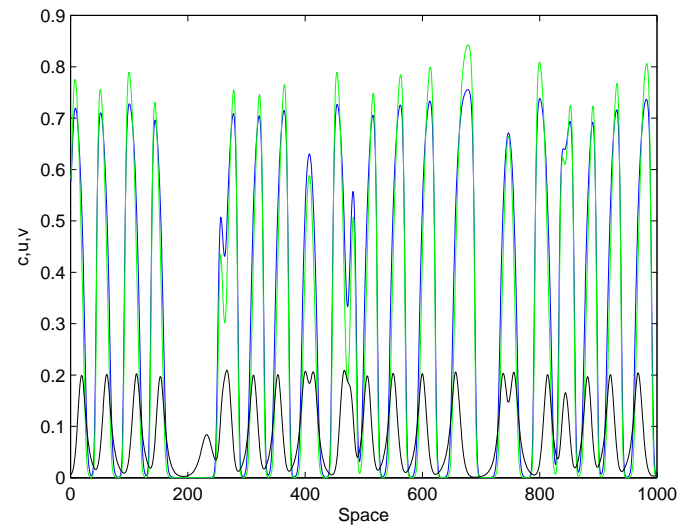

(b)

Figure 14: Snapshots of the oxygen and plankton spatial distribution obtained at (a) $t=200$ and (b) $t=2950$. $A_{0}=2.05$ and for $\omega=0.0001$, other parameters are given in the text. 
stable with regard to the changes in the oxygen production rate $A$ as the extinction of the corresponding nonspatial system occurred at $A \approx 2.055$ (cf. Figs. 7 and 9 ). However, a further increase in $A$ leads to the system crash (recall that in our model the global warming is assumed to continue indefinitely); in particular, for the parameters of Fig. 14, extinction occurs at $t \approx 4000$, i.e. for $A(t) \approx 2.4$. The corresponding temporal dynamics of the local and spatially averaged densities is shown in Fig. 15a and Fig. 15b, respectively. It is readily seen that, some time before the average values start their fast terminal decline, the amplitude of the irregular fluctuations decreases dramatically, which can be regarded as an early warning signal of the approaching catastrophe.

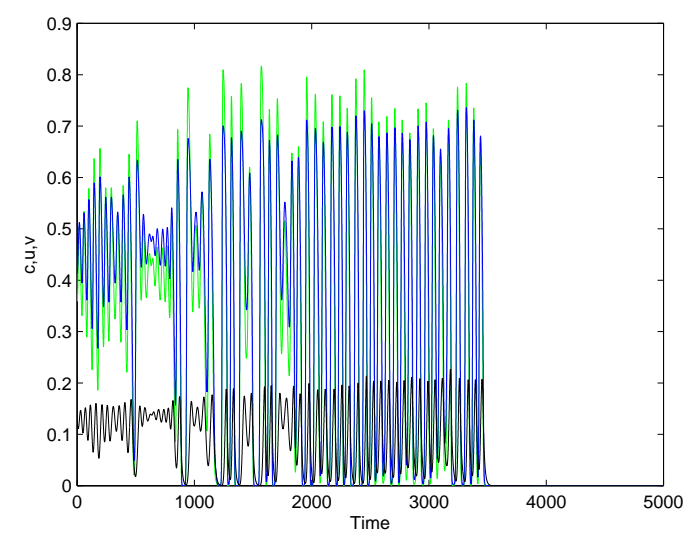

(a)

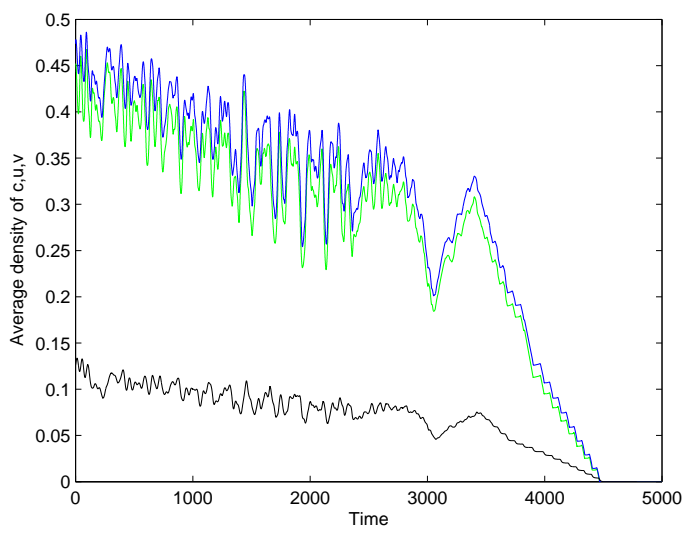

(b)

Figure 15: (a) System components distributions for fixed space $x_{0}=500$ for $t=5000$. (b) System components integration vs. time. The system parameters are taken from Figure $14 \mathrm{~b}$.

In conclusion to this section, we mention that the response of the spatial system to a decrease in $A$ (i.e. when the global warming hampers the oxygen production, cf. case (ii) in Section 4) appears to be similar to that of the nonspatial system. No pattern formation has been observed and the extinction occurs at approximately the same values of $A$ as in the nonspatial system.

\section{Discussion and Conclusions}

Potential effect of the global warming on the dynamics of populations, communities and ecosystems [15, 25, 51], terrestrial as well as aquatic [27, 63], has been an issue of a vigorous discussion recently. The focus of attention has usually been on the population dynamics to address the possibility of extinction or invasion of particular species [6, 14] and/or possible changes in the community structure [7, 49]. However, the overall effect 
of the climate change on ecosystems is likely to be much broader and is not exhausted by changes in species ranges and biodiversity loss. Indeed, ecosystems are not always subjects of passive response to large-scale environmental changes but may have active feedbacks. In particular, plankton systems are known to affect the climate on the global scale and hence to contribute to the climate change significantly [11, 78].

One aspect of the plankton systems functioning that remains poorly investigated is the effect of warming on the oxygen production by phytoplankton. Dynamics of the dissolved oxygen in marine ecosystems has been a focus on increasing attention recently $[2,8,10,42,43,58,75]$ as the concentration of dissolved oxygen is one of the fundamental environmental variables defining the well-being of a marine ecosystem. A decrease in the oxygen concentration below a certain level can results in hypoxia and mass mortality of marine fauna because of asphyxiation. Moreover, this is an issue of crucial importance not only for marine ecosystems themselves but also for terrestrial ecosystems on the global scale as about $70 \%$ of the atmospheric oxygen budget is produced in the oceans [33, 60]. However, the effect of water warming, in particular resulting from the global climate change, has not been considered by now. In this paper, we address this issue using mathematical modelling. It has been observed both in a few field studies as well as in some laboratory experiments that the overall rate of oxygen production by phytoplankton can change significantly as a result of an increase in the water temperature [32, 73]. Correspondingly, the goal of this study was to reveal the principle possibility of an ecological disaster, such as large-scale oxygen depletion, caused by such a change resulting from the global warming.

We have studied the oxygen-plankton dynamics using a mathematical model that takes into account oxygen production in photosynthesis, plankton respiration and the effect of zooplankton predation on phytoplankton; see Fig. 1. The model is described by a system of three coupled ODEs in the nonspatial (well-mixed) case and by three corresponding diffusion-reaction PDEs in the spatially explicit case. The system dynamics have been revealed by some analytical approaches and extensive numerical simulations. We first considered a nonspatial system to reveal the structure of the parameter space and showed that the system is sustainable only for intermediate values of the oxygen production rate. For a sufficiently low or sufficiently high oxygen production rate, plankton goes extinct and oxygen is depleted. For a baseline oxygen-phytoplankton system neglecting the presence of zooplankton, the system is sustainable unless the production rate becomes too low (see Fig. 2b). We then considered the dynamics of the corresponding non-autonomous system where the oxygen production rate slowly changes with time to take into account the increase in the water temperature due to the climate change. We showed that a sufficiently large increase or decrease in the production rate results is a bifurcation leading to a sudden depletion of oxygen and plankton extinction.

The corresponding spatially explicit system exhibits a complicated spatiotemporal dynamics typically resulting in the formation of a transient patchy pattern. Here we 
recall that plankton patchiness is a very common property of marine ecosystems [1, 23, 30, 52, 76] and the diffusion-reaction models of phyto-zooplankton dynamics have previously been used to describe this phenomenon, e.g. see [64], also [53, 54, 57] and references there. This self-organized pattern formation makes the system's dynamics sustainable in a somewhat broader range of oxygen production rate. However, a sufficiently large increase or decrease in the production rate leads to plankton extinction and oxygen depletion. Our main findings are summarized in the diagram shown in Fig. 16.

Our results have important implications. A lot has been said about detrimental consequences of the global warming such as, for instance, possible extinction of some species (and the corresponding biodiversity loss) and the large-scale flooding resulting from melting Antarctic ice. In this paper, however, we have shown that the danger to be stifled is probably more real than to be drowned. Using a model of coupled oxygen-plankton dynamics, we have identified another possible consequence of the global warming that can potentially be more dangerous than all others. We have shown that the oxygen production by marine phytoplankton can stop suddenly if the water temperature exceeds a certain critical value. Since the ocean plankton produces altogether more than one half of the total atmospheric oxygen, it would mean oxygen depletion not only in the water but also in the air. Should it happen, it would obviously kill most of life on Earth.

Note that it was not our aim here to calculate precise critical values of the oxygen production rate. Instead, our aim is to identify the new threat in principle rather than to link our analysis to specific plankton species or specific marine ecosystems. Simi-

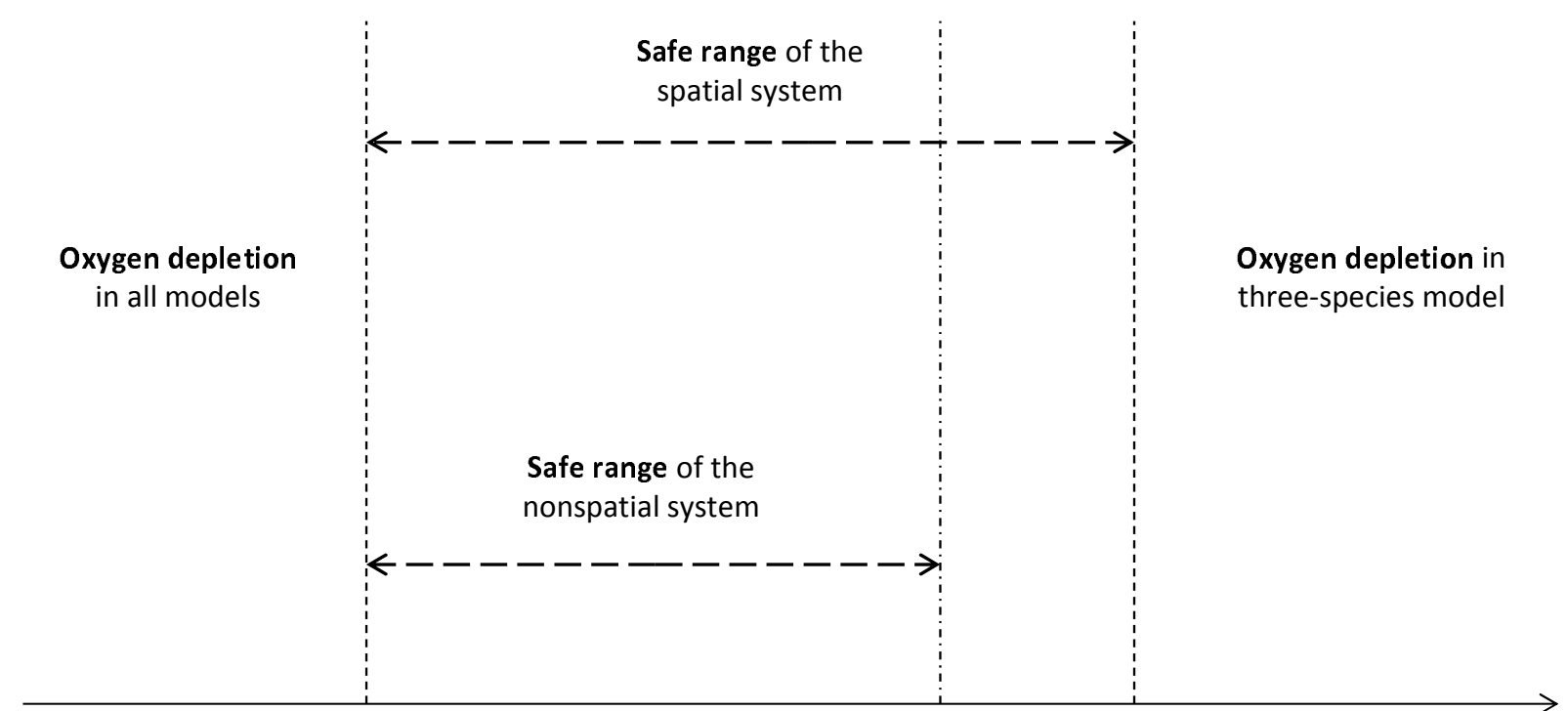

Low oxygen production rate

High oxygen production rate

Figure 16: Graphical summary of our main findings. 
larly, we do not attempt to estimate the value of the model parameters. In contrast to other simulation studies where complicated 'realistic' marine ecosystem models were used (e.g. [10, 24, 43]), our model is relatively simple. One benefit of this approach is that the model becomes tractable semi-analytically (see Section 3) and the change in model's properties in response to relevant factors can be revealed and understood relatively easy, cf. Section 3.1. Also, since our model takes into account only very general, generic interactions in the oxygen-plankton system, we believe that the model predictions possess a considerable degree of generality.

The downside of the model's simplicity is that many processes are left out of the scope. Thus, our study leaves a number of open questions. Firstly, in our model, the ocean hydrodynamics is taken into account very schematically (i.e. as the turbulent diffusion). However, the amount of oxygen entering the atmosphere through the ocean surface is known to depend on details of the ocean circulation [31, 36, 47, 55, 74]. Although it does not seem likely that ocean hydrodynamics can alter the dependence of oxygen production on temperature, it seems probable that it can delay the oxygen transport through the sea-air interface. How this delay may affect the atmospheric oxygen budget remains to be investigated. Secondly, our model does not take into account the effect of oxygen saturation and its dependence on temperature. Meanwhile, the solubility of oxygen in water is known to decline with water temperature, which can be another factor reducing the oxygen concentration [2, 36]. Thirdly, phytoplankton community consists of hundreds of different species. To the best of our knowledge, data on the temperature dependence of the oxygen production rate are currently available only for a handful of species [32, 73]. How general is the observed response to an increase in water temperature remains to be seen. Addressing these questions should become a subject of future research.

Acknowledgements. The authors are thankful to Nikolay Brilliantov (Leicester) for stimulating discussions at the early stage of this study. 


\section{Appendix}

The Jacobian matrix $J$, i.e. the matrix of the linearized system (19-21), is as follows:

$$
J=\left(\begin{array}{ccc}
-\frac{A u}{(1+c)^{2}}-1-\frac{u c_{2}}{\left(c+c_{2}\right)^{2}}-\frac{\nu v c_{3}}{\left(c+c_{3}\right)^{2}} & \frac{A}{1+c}-\frac{c}{c+c_{2}} & -\frac{\nu c}{c+c_{3}} \\
\frac{B c_{1} u}{\left(c+c_{1}\right)^{2}} & \frac{B c}{c+c_{1}}-2 u-\frac{v h}{(u+h)^{2}}-\sigma & -\frac{u}{u+h} \\
\frac{u v}{u+h} \frac{2 c c_{4}^{2}}{\left(c^{2}+c_{4}\right)^{2}} & \frac{v h}{(u+h)^{2}} \frac{c^{2}}{\left(c^{2}+c_{4}{ }^{2}\right)} & \frac{u c^{2}}{(u+h)\left(c^{2}+c_{4}{ }^{2}\right)}-\mu
\end{array}\right)
$$

Its specific form for each of the steady states is given below along with the corresponding characteristic equation.

- Extinction state $E_{1}=(0,0,0)$

The Jacobian matrix (39) takes the following form:

$$
J_{(0,0,0)}=\left(\begin{array}{ccc}
-1 & A & 0 \\
0 & -\sigma & 0 \\
0 & 0 & -\mu
\end{array}\right),
$$

and the characteristic equation is

$$
(1+\lambda)(\sigma+\lambda)(\mu+\lambda)=0
$$

- Zooplankton-free states $E_{2}^{(1)}$ and $E_{2}^{(1)}$

The Jacobian matrix:

$$
J_{(c, u, 0)}=\left(\begin{array}{ccc}
-\frac{A u}{(1+c)^{2}}-1-\frac{u c_{2}}{\left(c+c_{2}\right)^{2}}-\lambda & \frac{A}{c+1}-\frac{c}{c+c_{2}} & -\frac{\nu c}{c+c_{3}} \\
\frac{B c_{1} u}{\left(c+c_{1}\right)^{2}} & \frac{B c}{c+c_{1}}-2 u-\sigma-\lambda & -\frac{u}{u+h} \\
0 & 0 & \frac{u}{u+h} \frac{c^{2}}{c^{2}+c_{4}{ }^{2}}-\mu-\lambda
\end{array}\right)
$$

and the characteristic equation:

$$
\begin{aligned}
& {\left[\left(-\frac{A u}{(1+c)^{2}}-1-\frac{u c_{2}}{\left(c+c_{2}\right)^{2}}-\lambda\right)\left(\frac{B c}{c+c_{1}}-2 u-\sigma-\lambda\right)\right.} \\
& \left.-\left(\frac{A}{1+c}-\frac{c}{c+c_{2}}\right)\left(\frac{B c_{1} u}{\left(c+c_{1}\right)^{2}}\right)\right] \cdot\left(\frac{u}{u+h}\left(\frac{c^{2}}{c^{2}+c_{4}^{2}}\right)-\mu-\lambda\right)=0,
\end{aligned}
$$

where $c$ and $u$ are defined by Eqs. (26-27).

- Oxygen-phyto-zooplankton coexistence state $E_{3}$ 


$$
\begin{aligned}
& J_{(c, u, v)}= \\
& \left|\begin{array}{ccc}
-\frac{A u}{(1+c)^{2}}-1-\frac{u c_{2}}{\left(c+c_{2}\right)^{2}}-\frac{\nu v c_{3}}{\left(c+c_{3}\right)^{2}}-\lambda & \frac{A}{1+c}-\frac{c}{c+c_{2}} & -\frac{\nu c}{c+c_{3}} \\
\frac{B c_{1} u}{\left(c+c_{1}\right)^{2}} & \frac{B c}{c+c_{1}}-2 u-\frac{v h}{(u+h)^{2}}-\sigma-\lambda & -\frac{u}{u+h} \\
\frac{u v}{u+h} \frac{2 c c_{4}{ }^{2}}{\left(c^{2}+c_{4}\right)^{2}} & \frac{v h}{(u+h)^{2}} \frac{c^{2}}{\left(c^{2}+c_{4}{ }^{2}\right)} & \frac{u c^{2}}{(u+h)\left(c^{2}+c_{4}{ }^{2}\right)}-\mu-\lambda
\end{array}\right|
\end{aligned}
$$

where $c, u$ and $v$ are defined by Eqs. (28 30). 


\section{References}

[1] Abbott MR (1993) Phytoplankton patchiness: ecological implications and observation methods. In Levin SA, Powell TM, Steele JH (eds.). Patch dynamics. Lecture Notes in Biomathematics, Vol. 96. Springer, Berlin 37-49

[2] Addy, K. and Green, L. (1997). Dissolved oxygen and temperature. Fact Sheet No. 963, Natural Resources Facts, University of Rhodes Island.

[3] Allegretto W, Mocenni C, Vicino A (2005) Periodic solutions in modelling lagoon ecological interactions. J Math Biol 51:367-388

[4] Andersson A, Haecky P, Hagstrom A (1994) Effect of temperature and light on the growth of micro- nano- and pico-plankton: impact on algal succession. Mar Biol $120: 511-520$

[5] Behrenfeld MJ, Falkowski PG (1997) A consumers guide to phytoplankton primary productivity models. Limnol Oceanogr 42:1479-1491

[6] Berestycki H, Desvillettes L, Diekmann O (2014) Can climate change lead to gap formation? Ecol Compl 20:264-270

[7] Bonnefon O, Coville J, Garnier J, Hamel F, Roques L (2014) The spatio-temporal dynamics of neutral genetic diversity. Ecol Compl 20:282-292

[8] Breitburg DL, Loher T, Pacey CA, Gerstein A (1997) Varying effects of low dissolved oxygen on trophic interactions in an estuarine food web. Ecol Monogr 67:489-507

[9] Carpenter JH (1966) New measurements of oxygen solubility in pure and natural water. Limnol Oceanogr 11:264-277

[10] Chapelle A, Ménesguen A, Deslous-Paoli J-M et al (2000) Modelling nitrogen, primary production and oxygen in a Mediterranean lagoon: impact of oysters farming and inputs from the watershed. Ecol Model 127:161-181

[11] Charlson RJ, Lovelock JE, Andreae MO, Warren SG (1987) Oceanic phytoplankton, atmospheric sulphur, cloud albedo and climate, Nature 326:655-661

[12] Childress JJ (1975) The respiratory rates of midwater crustaceans as a function of depth of occurrence and relation to the oxygen minimum layer of Southern California. Compar Biochem Physiol Part A: Physiology 50:787-799

[13] Childress JJ (1976) Effects of pressure, temperature and oxygen on the oxygen consumption rate of the midwater copepod Gaussia princeps. Mar Biol 39:19-24 
[14] Cosner C (2014) Challenges in modeling biological invasions and population distributions in a changing climate. Ecol Compl 20:258-263

[15] Culos GJ, Tyson RC (2014) Response of poikilotherms to thermal aspects of climate change. Ecol Compl 20:293-306

[16] Cushing DH (1975) Marine ecology and fisheries. Cambridge University Press, Cambridge

[17] Davenport J, Trueman ER (1985) Oxygen uptake and buoyancy in zooplanktonic organisms from the tropical Eastern Atlantic. Compar Biochem Physiol Part A: Physiology 81:857-863

[18] Decker MB, Breitburg DL, Purcell JE (2004) Effects of low dissolved oxygen on zooplankton predation by the ctenophore Mnemiopsis leidyi. Mar Ecol Progr Ser 280:163-172

[19] Denman K, Hofmann E, Marchant H (1996) Marine biotic responses and feedbacks to environmental change and feedbacks to climate. in Houghton JT, et al. (eds.). Climate Change 1995. The Science of Climate Change. Cambridge University Press, Cambridge, 483-516

[20] Devol AH (1981) Vertical distribution of zooplankton respiration in relation to the intense oxygen minimum zones in two British Columbia fjords. J Plankt Res 3:593602

[21] Enquist BJ, Economo EP, Huxman TE et al (2003) Scaling metabolism from organisms to ecosystems. Nature 423:639-642

[22] Eppley RW (1972) Temperature and phytoplankton growth in the sea. Fish Bull 70:1063-1085

[23] Fasham M (1978) The statistical and mathematical analysis of plankton patchiness. Oceanogr Mar Biol Ann Rev 16:43-79

[24] Fasham MJR, Ducklow HW, McKelvie SM (1990) A nitrogen-based model of plankton dynamics in the oceanic mixed layer. J Marine Res 48:591-639

[25] Ferrarini A, Rossi G, Mondoni A, Orsenigo S (2014) Prediction of climate warming impacts on plant species could be more complex than expected. Evidence from a case study in the Himalaya. Ecol Compl 20:307-314

[26] Franke U, Hutter K, Johnk K (1999) A physical-biological coupled model for algal dynamics in lakes. Bull Math Biol 61:239-272 
[27] Franssen SU, Gu J, Bergmann N et al (2011) Transcriptomic resilience to global warming in the seagrass Zostera marina, a marine foundation species. Proc Natl Acad Sci USA 108:19276-19281

[28] Garcia HE, Gordon LI (1992). Oxygen solubility in seawater: better fitting equations. Limnol Oceanogr 37:1307-1312

[29] Gliwicz MZ (1986) Predation and the evolution of vertical migration in zooplankton. Nature 320:746-748

[30] Greene CH, Widder EA, Youngbluth MJ, Tamse A, Johnson GE (1992) The migration behavior, fine structure, and bioluminescent activity of krill sound-scattering layers. Limnol Oceanogr 37:650-658

[31] Hamme RC, Keeling RF (2008) Ocean ventilation as a driver of interannual variability in atmospheric potential oxygen. Tellus B 60:706-717

[32] Hancke K, Glud RN (2004) Temperature effects on respiration and photosynthesis in three diatom-dominated benthic communities. Aquat Microb Ecol 37:265-281

[33] Harris GP (1986) Phytoplankton ecology: structure, function and fluctuation. Springer, Berlin

[34] Hastings A (2001) Transient dynamics and persistence of ecological systems. Ecol Lett $4: 215-220$

[35] Hastings A (2004) Transients: the key to long-term ecological understanding? Trends Ecol Evolut 19:39-45

[36] Hein B, Viergutz C, Wyrwa J, Kirchesch V, Schöl A (2014) Modelling the impact of climate change on phytoplankton dynamics and the oxygen budget of the Elbe river and estuary (Germany). In Lehfeldt R, Kopmann R (eds.). Hamburg, ICHE 2014 $1035-1042$

[37] Hernandez-Leon S, Ikeda T (2005) Zooplankton respiration: respiration in aquatic systems. Oxford University Press, New York

[38] Hoppe H-G, Gocke K, Koppe R, Begler C (2002) Bacterial growth and primary production along a North-South transect of the Atlantic Ocean. Nature 416:168-171

[39] Huisman J, Weissing FJ (1995) Competition for nutrients and light in a mixed water column: a theoretical analysis. Amer Nat 146:536-564

[40] Huisman J, van Oostveen P, Weissing FJ (1999) Species dynamics in phytoplankton blooms: incomplete mixing and competition for light. Amer Nat 154:46-68 
[41] Huisman J, Sharples J, Stroom JM, Visser PM, Kardinaal WEA, Verspa JM, Sommeijer B (2004) Changes in turbulent mixing shift competition for light between phytoplankton species. Ecology 85:2960-2970

[42] Hull V, Mocenni C, Falcucci M, Marchettini N (2000) A trophodynamic model for the Lagoon of Fogliano (Italy) with ecological dependent modifying parameters. Ecol Model 134:153-167

[43] Hull V, Parrella L, Falcucci M (2008) Modelling dissolved oxygen dynamics in coastal lagoons. Ecol Model 211:468-480

[44] Intergovernmental Panel on Climate Change (2014). Climate change 2014: synthesis report. Contribution of Working Groups I, II and III to the Fifth Assessment Report of the Intergovernmental Panel on climate change (Core Writing Team, Pachauri RK, Meyer LA, eds.). IPCC, Geneva

[45] Jin Z, Charlock TP, Smith WL, Rutledge K (2004) A parametrization of ocean surface albedo. Geophys Res Lett 31:L22301

[46] Jones RI (1977) The importance of temperature conditioning to the respiration of natural phytoplankton communities. Brit Phycological J 12:277-285

[47] Keeling R F, Kortzinger A, Gruber N (2010) Ocean deoxygenation in a warming world. Mar Sci 2:199-229

[48] Kremer J, Nixon SW (1978) A coastal marine ecosystem: simulation and analysis. Springer, Berlin

[49] Kyriazopoulos P, Nathan J, Meron E (2014) Species coexistence by front pinning. Ecol Compl 20:271-281

[50] Li W, Smith J, Platt T (1984) Temperature response of photosynthetic capacity and carboxylase activity in arctic marine phytoplankton. Mar Ecol Progr Ser 17:237-243

[51] Long A, Tyson RC (2014) Integrating Homo sapiens into ecological models: imperatives of climate change. Ecol Compl 20:325-334

[52] Mackas DL, Boyd CM (1979) Spectral analysis of zooplankton spatial heterogeneity. Science 204:62-64

[53] Malchow H, Petrovskii SV, Hilker FM (2003) Models of spatiotemporal pattern formation in plankton dynamics. Nova Acta Leopoldina NF 88:325-340

[54] Malchow H, Petrovskii SV, Venturino E (2008) Spatiotemporal patterns in ecology and epidemiology: theory, models, and simulation. CRC Press, Boca Raton 
[55] Matear R, Hirst A, McNeil B (2000) Changes in dissolved oxygen in the Southern Ocean with climate change. Geochem Geophys Geosyst 1(11)

[56] Martin AP (2003) Phytoplankton patchiness: the role of lateral stirring and mixing. Progr Oceanogr 57:125-174

[57] Medvinsky AB, Petrovskii SV, Tikhonova IA, Malchow H, Li B-L (2002). Spatiotemporal complexity of plankton and fish dynamics. SIAM Rev 44:311-370

[58] Misra A (2010) Modeling the depletion of dissolved oxygen in a lake due to submerged macrophytes. Nonlin Anal Model Cont 15:185-198

[59] Monin AS, Yaglom AM (1971) Statistical fluid mechanics: mechanics of turbulence. Vol. 1. MIT Press, Cambridge MA

[60] Moss BR (2009) Ecology of fresh waters: man and medium, past to future. John Wiley \& Sons, London

[61] Najjar RG, Walker HA, Anderson PJ et al (2000). The potential impacts of climate change on the mid-Atlantic coastal region. Climate Res 14:219-233

[62] Najjar RG, Pyke CR, Adams MB et al (2010) Potential climate-change impacts on the Chesapeake Bay. Estuar Coastal Shelf Sci 86:1-20

[63] Nguyen KDT, Morley SA, La CH et al (2011) Upper temperature limits of tropical marine ectotherms: global warming implications. PLoS ONE 6(12):e29340

[64] Okubo A (1980) Diffusion and ecological problems: mathematical models. SpringerVerlag, Berlin

[65] Petrovskii SV, Malchow H (2001) Wave of chaos: new mechanism of pattern formation in spatio-temporal population dynamics. Theor Popul Biol 59:157-174

[66] Petrovskii SV, Malchow H (2004) Mathematical models of marine ecosystems. In: The Encyclopedia of Life Support Systems (EOLSS), EOLSS Publishers, Oxford UK

[67] Petrovskii SV, Li B-L, Malchow H (2004) Transition to spatiotemporal chaos can resolve the paradox of enrichment. Ecol Compl 1:37-47

[68] Petrovskii SV, Morozov AY, and Venturino E (2002) Allee effect makes possible patchy invasion in a predator-prey system. Ecol Lett 5:345-352

[69] Petrovskii SV, Kawasaki K, Takasu F, Shigesada N (2001) Diffusive waves, dynamical stabilization and spatio-temporal chaos in a community of three competitive species. Japan J Industr Appl Maths 18:459-481 
[70] Pinel-Alloul B (1995) Spatial heterogeneity as a multiscale characteristic of zooplankton community, Hydrobiologia 300/301:17-42

[71] Prosser CL (1961) Oxygen: respiration and metabolism. In Prosser CL, Brown FA (eds). Comparative animal physiology. WB Saunders, Philadelphia, PA 165-211

[72] Raven JA, Geider RJ (1988). Temperature and algal growth. New Phytol 110:441-461

[73] Robinson C (2000) Plankton gross production and respiration in the shallow water hydrothermal systems of Milos, Aegean Sea. J Plankt Res 22:887-906

[74] Shaffer G, Leth O, Ulloa O et al (2000) Warming and circulation change in the Eastern South Pacific Ocean. Geophys Res Lett 27:1247-1250

[75] Sekerci Y, Petrovskii S (2015) Mathematical modelling of spatiotemporal dynamics of oxygen in a plankton system. Math Model Nat Phenom 7:96-114

[76] Steele JH (1978) Spatial pattern in plankton communities. Plenum, London

[77] Steel JA (1980) Phytoplankton models. In LeCren ED, Lowe-McConnell RH (eds.). Functioning of freshwater ecosystems, Vol. 2. Cambridge University Press, Cambridge $220-227$

[78] Williamson P, Gribbin J (1991) How plankton change the climate. New Scientist, March 16, 1991, 48-52 